\title{
An evaluation of the exposure in nadir observation of the JEM-EUSO mission
}

\begin{abstract}
J.H. Adams Jr. ${ }^{m d}$, S. Ahmad ${ }^{b a}$, J.-N. Albert ${ }^{b a}$, D. Allard ${ }^{b b}$, M. Ambrosio ${ }^{d f}$, L. Anchordoqui ${ }^{m e}$, A. Anzalone ${ }^{d h}$, Y. Arai ${ }^{e u}$, C. Aramo ${ }^{d f}$, K. Asano ${ }^{e s}$, M. Ave ${ }^{k f}$, P. Barrillon ${ }^{b a}$, T. Batsch ${ }^{h c}$, J. Bayer $^{c d}$, T. Belenguer ${ }^{k b}$, R. Bellotti ${ }^{d b}$, A.A. Berlind ${ }^{m g}$, M. Bertaina ${ }^{d l, d k, 1}$, P.L. Biermann ${ }^{c b}$, S. Biktemerova ${ }^{i a}$, C. Blaksley ${ }^{b b}$, J. Błęcki ${ }^{h e}, \mathrm{~S}$. Blin-Bondil $^{b a}$, J. Blümer ${ }^{c b}$, P. Bobik ${ }^{j a}$, M. Bogomilov ${ }^{a a}$, M. Bonamente ${ }^{m d}$, M.S. Briggs ${ }^{m d}$, S. Briz ${ }^{k e}$, A. Bruno $^{d a}$, F. Cafagna ${ }^{d a}$, D. Campana ${ }^{d f}$, J-N. Capdevielle ${ }^{b b}$, R. Caruso ${ }^{d c}$, M. Casolino ${ }^{e v, d i, d j}$, C. Cassardo ${ }^{d l, d k}$, G. Castellini ${ }^{d d}$, O. Catalano ${ }^{d h}$, A. Cellino ${ }^{d m, d k}$, M. Chikawa ${ }^{e d}$, M.J. Christl ${ }^{m f}$, V. Connaughton ${ }^{m d}$,
\end{abstract}

J.F. Cortés ${ }^{k e}$, H.J. Crawford ${ }^{m a}$, R. Cremoninid ${ }^{d l}$, S. Csorna ${ }^{m g}$, J.C. D'Olivo ${ }^{g a}$, S. Dagoret-Campagne ${ }^{b a}$, A.J. de Castro ${ }^{k e}$, C. De Donato ${ }^{d i, d j}$, C. de la Taille ${ }^{b a}$,

L. del Peral ${ }^{k d}$, A. Dell'Oro ${ }^{d m, d k}$ M.P. De Pascale ${ }^{d i, d j}$, M. Di Martino ${ }^{d m, d k}$,

G. Distratis ${ }^{c d}$, M. Dupieux ${ }^{b c}$, A. Ebersoldt ${ }^{c b}$, T. Ebisuzaki ${ }^{e v}$, R. Engel ${ }^{c b}$,

S. Falk ${ }^{c b}$, K. Fang ${ }^{m b}$, F. Fenu ${ }^{c d}$, I. Fernández-Gómez ${ }^{k e}$, S. Ferrarese ${ }^{d l, d k}$,

A. Franceschi ${ }^{d e}$, J. Fujimoto ${ }^{e u}$, P. Galeotti ${ }^{d l, d k}$, G. Garipov ${ }^{i c}$, J. Geary ${ }^{m d}$, U.G. Giaccari ${ }^{d f}$, G. Giraudo ${ }^{d k}$, M. Gonchar ${ }^{i a}$, C. González Alvarado ${ }^{k b}$, P. Gorodetzky ${ }^{b b}$, F. Guarino ${ }^{d f, d g}$, A. Guzmán ${ }^{c d}$, Y. Hachisu ${ }^{e v}$, B. Harlov ${ }^{i b}$, A. Haungs ${ }^{c b}$, J. Hernández Carretero ${ }^{k d}$, K. Higashide ${ }^{e q, e v}$, T. Iguchi ${ }^{e i}$, H. Ikeda ${ }^{e o}, \mathrm{~N}$. Inoue $^{e q}, \mathrm{~S}$. Inoue ${ }^{e t}, \mathrm{~A}$. Insolia ${ }^{d c}, \mathrm{~F}$. Isgrò ${ }^{d f, d g}$, Y. Itow $^{e n}$, E. Joven ${ }^{k g}$, E.G. Judd ${ }^{m a}$, A. Jung ${ }^{f c}$, F. Kajino ${ }^{e i}$, T. Kajino ${ }^{e l}$, I. Kaneko ${ }^{e v}$, Y. Karadzhov ${ }^{a a}$, J. Karczmarczyk ${ }^{h c}$, K. Katahira ${ }^{e v}$, K. Kawai ${ }^{e v}$, Y. Kawasaki ${ }^{e v}$, B. Keilhauer ${ }^{c b}$, B.A. Khrenov ${ }^{i c}$, Jeong-Sook Kim ${ }^{f b}$, Soon-Wook Kim ${ }^{f b}$, Sug-Whan Kim ${ }^{f d}$, M. Kleifges ${ }^{c b}$, P.A. Klimov ${ }^{i c}$, S.H. Ko ${ }^{f a}$, D. Kolev ${ }^{a a}$, I. Kreykenbohm ${ }^{c a}$, K. Kudela ${ }^{j a}$, Y. Kurihara ${ }^{e u}$, E. Kuznetsov ${ }^{m d}$, G. La Rosa ${ }^{d h}$, J. Lee ${ }^{f c}$, J. Licandro ${ }^{k g}$, H. Lim ${ }^{f c}$, F. López ${ }^{k e}$, M.C. Maccarone ${ }^{d h}$, K. Mannheim ${ }^{c e}$, L. Marcellidi,dj, A. Marini ${ }^{d e}$, G. Martin-Chassard ${ }^{b a}$, O. Martinez ${ }^{g c}$, G. Masciantonio ${ }^{d i, d j}$, K. Mase $^{e a}$, R. Matev ${ }^{a a}$, A. Maurissen ${ }^{l a}$, G. Medina-Tanco ${ }^{g a}$, T. Mernik ${ }^{c d}$, H. Miyamoto ${ }^{e v}$, Y. Miyazaki ${ }^{e c}$, Y. Mizumoto ${ }^{e l}$, G. Modestino ${ }^{d e}$, D. Monnier-Ragaigne ${ }^{b a}$, J.A. Morales de los Ríos ${ }^{k d}$, B. Mot ${ }^{b c}$, T. Murakamief ${ }^{e f}$ M. Nagano ${ }^{e c}$, M. Nagata ${ }^{e h}$, S. Nagataki ${ }^{e k}$, T. Nakamura ${ }^{e j}$, J.W. Nam ${ }^{f c}$,

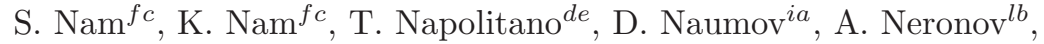
K. Nomoto ${ }^{e t}$, T. Ogawa ${ }^{e v}$, H. Ohmori ${ }^{e v}$, A.V. Olinto ${ }^{m b}$, P. Orleański ${ }^{h e}$, G. Osteria ${ }^{d f}$, N. Pacheco ${ }^{k c}$, M.I. Panasyuk ${ }^{i c}$, E. Parizot ${ }^{b b}$, I.H. Park ${ }^{f c}$, B. Pastircak ${ }^{j a}$, T. Patzak ${ }^{b b}$, T. Paul ${ }^{m e}$, C. Pennypacker ${ }^{m a}$, T. Peter ${ }^{l c}$, P. Picozza ${ }^{d i, d j, e v}$, A. Pollini ${ }^{l a}$, H. Prieto ${ }^{k d, k a}$, P. Reardon ${ }^{m d}$, M. Reina ${ }^{k b}$, M. Reyes ${ }^{k g}$, M. Ricci ${ }^{d e}$, I. Rodríguez ${ }^{k e}$, M.D. Rodríguez Frías ${ }^{k d}$, F. Ronga ${ }^{d e}$, H. Rothkaehl ${ }^{h e}$, G. Roudil ${ }^{b c}$, I. Rusinov ${ }^{a a}$, M. Rybczyński ${ }^{h a}$, M.D. Sabau ${ }^{k b}$, G. Sáez Cano ${ }^{k d}$, A. Saito ${ }^{e j}$, N. Sakaki ${ }^{c b}$, M. Sakata ${ }^{e i}$, H. Salazar ${ }^{g c}$, S. Sánchez ${ }^{k e}$, A. Santangelo ${ }^{c d}$, L. Santiago Crúz ${ }^{g a}$, M. Sanz Palomino ${ }^{k b}$, O. Saprykin ${ }^{i b}$, F. Sarazin ${ }^{m c}$, H. Sato ${ }^{e i}$, M. Sato ${ }^{e r}$, T. Schanz ${ }^{c d}$, H. Schieler ${ }^{c b}$, V. Scotti ${ }^{d f}, d g$, M. Scuderid ${ }^{d c}$, A. Segreto ${ }^{d h}$, S. Selmane ${ }^{b b}$, D. Semikoz ${ }^{b b}$, 
M. Serra ${ }^{k g}$, S. Sharakin ${ }^{i c}$, T. Shibata ${ }^{e p}$, H.M. Shimizu ${ }^{e m}$, K. Shinozaki ${ }^{e v, 1}$, T. Shirahama ${ }^{e q}$, G. Siemieniec-Oziębło ${ }^{h b}$, H.H. Silva López ${ }^{g a}$, J. Sledd ${ }^{m f}$, K. Słomińska ${ }^{h e}$, A. Sobey ${ }^{m f}$, T. Sugiyama ${ }^{e m}$, D. Supanitsky ${ }^{g a}$, M. Suzuki ${ }^{e o}$, B. Szabelska ${ }^{h c}$, J. Szabelski ${ }^{h c}$, F. Tajima ${ }^{e e}$, N. Tajima ${ }^{e v}$, T. Tajima ${ }^{c c}$, Y. Takahashi ${ }^{e r}$, H. Takami $^{e u}$, M. Takeda ${ }^{e g}$, Y. Takizawa ${ }^{e v}$, C. Tenzer ${ }^{c d}$, O. Tibolla ${ }^{c e}, \mathrm{~L}$. Tkachev ${ }^{i a}, \mathrm{~T}$. Tomida ${ }^{e v}, \mathrm{~N}$. Tone ${ }^{e v}, \mathrm{~F}$. Trillaud ${ }^{g a}$,

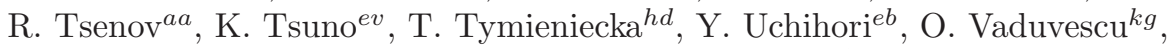
J.F. Valdés-Galicia ${ }^{g a}$, P. Vallania ${ }^{d m, d k}$, L. Valore ${ }^{d f}$, G. Vankova ${ }^{a a}$,

C. Vigorito ${ }^{d l, d k}$, L. Villaseñor ${ }^{g b}$, P. von Ballmoos ${ }^{b c}$, S. Wada ${ }^{e v}$, J. Watanabe $^{e l}$, S. Watanabe ${ }^{e r}$, J. Watts Jr. ${ }^{m d}$, M. Weber ${ }^{c b}$, T.J. Weiler ${ }^{m g}$, T. Wibig ${ }^{h c}$, L. Wiencke ${ }^{m c}$, M. Wille ${ }^{c a}$, J. Wilms ${ }^{c a}$, Z. Włodarczyk ${ }^{h a}$, T. Yamamoto ${ }^{e i}$, Y. Yamamoto ${ }^{e i}$, J. Yang ${ }^{f c}$, H. Yano ${ }^{e o}$, I.V. Yashin ${ }^{i c}$, D. Yonetoku ${ }^{e f}$, K. Yoshida ${ }^{e i}$, S. Yoshida ${ }^{e a}$, R. Young ${ }^{m f}$, A. Zamora ${ }^{g a}$, A. Zuccaro Marchi $^{e v}$

a St. Kliment Ohridski University of Sofia, Bulgaria

ba Laboratoire de l'Accélérateur Linéaire, Univ Paris Sud-11, CNES/IN2P3, Orsay, France ${ }^{b b}$ APC, Univ Paris Diderot, CNRS/IN2P3, CEA/Irfu, Obs de Paris, Sorbonne Paris Cité, France
bc IRAP, Université de Toulouse, CNRS, Toulouse, France

ca ECAP, University of Erlangen-Nuremberg, Germany

cb Karlsruhe Institute of Technology (KIT), Germany

cc Ludwig Maximilian University, Munich, Germany

${ }^{c d}$ Institute for Astronomy and Astrophysics, Kepler Center, University of Tübingen, Germany

ce Institute for Theoretical Physics and Astrophysics, University of Würzburg, Germany da Istituto Nazionale di Fisica Nucleare - Sezione di Bari, Italy

db Universita'degli Studi di Bari Aldo Moro and INFN - Sezione di Bari, Italy

dc Dipartimento di Fisica e Astronomia - Universita' di Catania, Italy

dd Consiglio Nazionale delle Ricerche - Istituto Nazionale di Ottica Firenze, Italy

de Istituto Nazionale di Fisica Nucleare - Laboratori Nazionali di Frascati, Italy

df Istituto Nazionale di Fisica Nucleare - Sezione di Napoli, Italy

dg Universita' di Napoli Federico II - Dipartimento di Scienze Fisiche, Italy

dh INAF - Istituto di Astrofisica Spaziale e Fisica Cosmica di Palermo, Italy

di Istituto Nazionale di Fisica Nucleare - Sezione di Roma Tor Vergata, Italy

dj Universita' di Roma Tor Vergata - Dipartimento di Fisica, Roma, Italy

${ }^{d k}$ Istituto Nazionale di Fisica Nucleare - Sezione di Torino, Italy

dl Dipartimento di Fisica, Universita' di Torino, Italy

dm Osservatorio Astrofisico di Torino, Istituto Nazionale di Astrofisica, Italy

ea Chiba University, Chiba, Japan

eb National Institute of Radiological Sciences, Chiba, Japan

ec Fukui University of Technology, Fukui, Japan

ed Kinki University, Higashi-Osaka, Japan

ee Hiroshima University, Hiroshima, Japan

ef Kanazawa University, Kanazawa, Japan

eg Institute for Cosmic Ray Research, University of Tokyo, Kashiwa, Japan

eh Kobe University, Kobe, Japan

ei Konan University, Kobe, Japan

ej Kyoto University, Kyoto, Japan

ek Yukawa Institute, Kyoto University, Kyoto, Japan

el National Astronomical Observatory, Mitaka, Japan em Nagoya University, Nagoya, Japan

en Solar-Terrestrial Environment Laboratory, Nagoya University, Nagoya, Japan

eo Institute of Space and Astronautical Science/JAXA, Sagamihara, Japan

ep Aoyama Gakuin University, Sagamihara, Japan

eq Saitama University, Saitama, Japan 


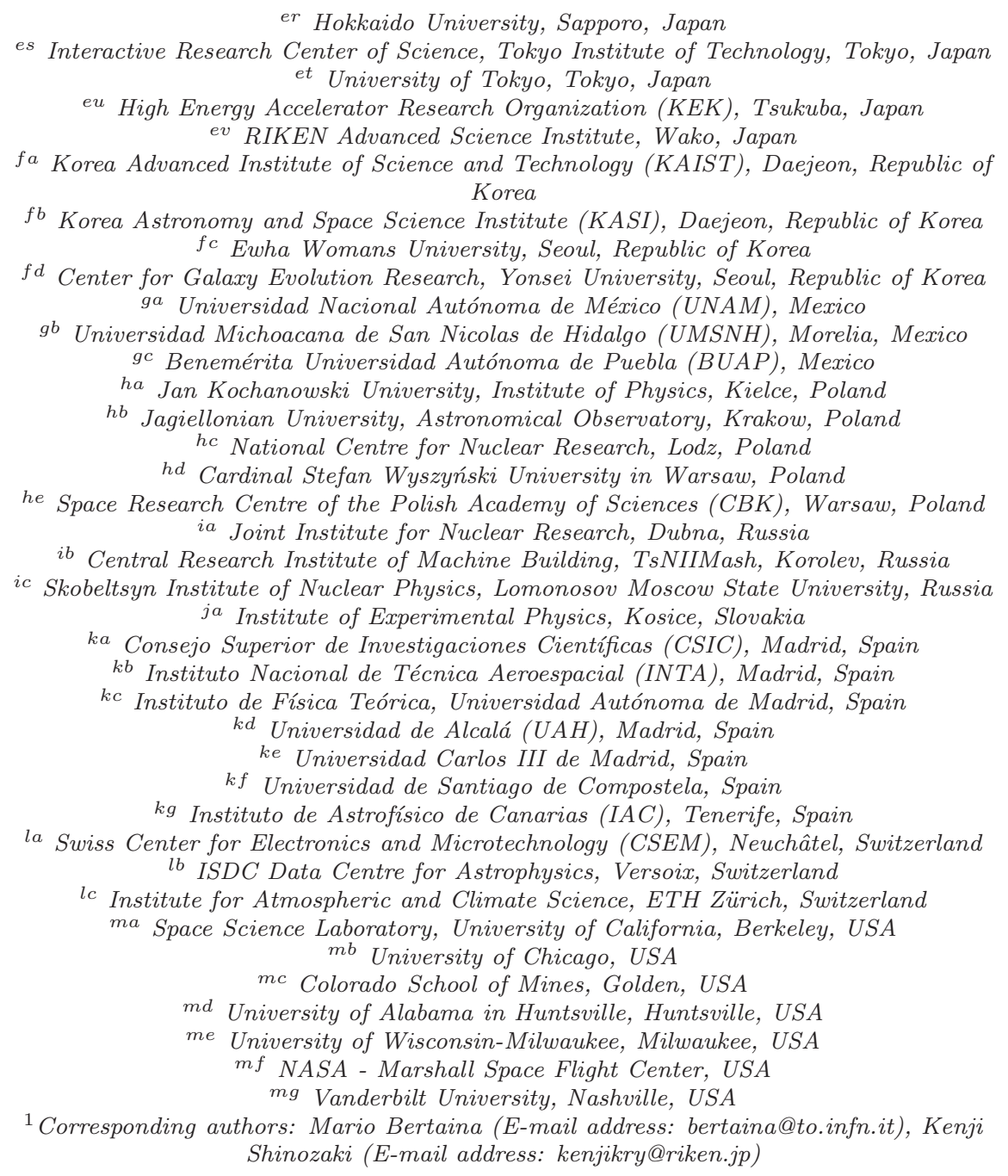

\begin{abstract}
We evaluate the exposure during nadir observations with JEM-EUSO, the Extreme Universe Space Observatory, on-board the Japanese Experiment Module of the International Space Station. Designed as a mission to explore the extreme energy Universe from space, JEM-EUSO will monitor the Earth's nighttime atmosphere to record the ultraviolet light from tracks generated by extensive air showers initiated by ultra-high energy cosmic rays. In the present work, we discuss the particularities of space-based observation and we compute the annual
\end{abstract}


exposure in nadir observation. The results are based on studies of the expected trigger aperture and observational duty cycle, as well as, on the investigations of the effects of clouds and different types of background light. We show that the annual exposure is about one order of magnitude higher than those of the presently operating ground-based observatories.

\section{Introduction}

The origin and nature of Ultra-High Energy Cosmic Rays (UHECRs) remains unsolved in contemporary astroparticle physics. Possible indications of sources or excesses in the arrival direction distribution of UHECRs have been claimed by ground-based experiments, though not fully confirmed [1, 2, 3]. In order to be identified from Earth, extremely powerful sources capable of accelerating cosmic rays up to ultra-high energies must be within a limited range of distances set by the Greisen-Zatseptin-Kuz'min (GZK) effect [4, 5].

Since the distribution of matter within the GZK range is inhomogeneous and anisotropic, one would expect UHECR arrival directions to exhibit a corresponding anisotropy. To identify the sources of UHECRs, measurements of the energy spectrum and arrival directions with high statistics are essential. This is rather challenging because of the extremely low flux of a few per $\mathrm{km}^{2}$ per century at extreme energies such as $E>5 \times 10^{19} \mathrm{eV}$. The observational exposure is, therefore, a critical factor.

JEM-EUSO (the Extreme Universe Space Observatory on-board the Japanese Experiment Module) [6, 7, 8] on the International Space Station (ISS) 9] is an innovative space mission. Looking down the Earth from space, it utilizes the atmosphere as a detector of cosmic ray air showers with the aim of significantly increasing the exposure to UHECRs compared to the largest ground-based air shower arrays presently in operation 10, 11, 12]. The JEM-EUSO telescope will be accommodated on the Exposed Facility (EF) on the JEM module Kibo 13] of the ISS. The scientific objectives include charged particle astronomy and astrophysics, as well as, other exploratory objectives [14] such as the detection of extreme energy gamma rays and neutrinos. The JEM-EUSO telescope exploits the fluorescence light that is emitted during the development of the Extensive Air Shower (EAS), initiated by a primary cosmic ray particle in the atmosphere to estimate the particle's energy and arrival direction. This is an established technique that has been employed by several ground-based UHECR observatories [10, 12, 15, 16], but never in space-based observations.

The estimation of the exposure of a space-based experiment such as JEMEUSO requires accounting for: a) the characteristics of the EAS development in the atmosphere as observed from space, b) the properties of the telescope, including its orbit and Field of View (FoV), c) the various sources of steady background like night-glow and moonlight, d) the overall optical transmission properties of the atmosphere, in particular the possible presence of clouds, and e) the effect of anthropogenic light, atmospheric flashes such as Transient Luminous Events (TLEs) and meteors. Items a) and b) are the principal factors 
determining the threshold in energy and maximum aperture of the telescope. Item c) limits the observational duty cycle of the mission. Items d) and e) affect the instantaneous aperture of the telescope. In the following sections, all of these aspects will be reviewed.

The outline of this article is as follows: in Section 2, we summarize the key aspects of the JEM-EUSO mission. In Sections 3 and 4 , we discuss the estimation of the observational duty cycle, local light effects and the role of clouds. Section 5 is devoted to the trigger architecture and EAS simulation. Computation of the aperture for both clear and cloudy conditions will be described in Section 6 The exposure in the nadir observation and its uniformity will then be derived in Section 7 The paper concludes with a discussion of the results and a summary in Sections 8 and 9

\section{JEM-EUSO telescope and its observation principle}

The JEM-EUSO telescope [17] consists of four principal parts: the photon collecting optics [18], the Focal Surface (FS) detector [19], the electronics [20], and the mechanical structure [21]. The main parameters of JEM-EUSO telescope are summarized in Table 1. The telescope optics consists of three double-sided curved circular Fresnel lenses with $2.65 \mathrm{~m}$ maximum diameter. The minimum diameter of the lenses is $1.9 \mathrm{~m}$ owing to cuts on opposite sides. This shape is referred to as 'side-cut' and is required to satisfy constraints of the H-IIB Transfer Vehicle (HTV) Kounotori 13] which will transport the JEM-EUSO telescope to the ISS. The UV photons are focused onto the FS which consists of 137 Photo-Detector Modules (PDMs). Each PDM comprises of a $3 \times 3$ set of Elementary Cells (ECs). Each EC is formed by a $2 \times 2$ array of Multi-Anode PhotoMultiplier Tubes (MAPMTs) - Hamamatsu Photonics K.K. R11265-03-M64 - with $8 \times 8(=64)$ pixels. Each pixel has a spatial resolution of $0.074^{\circ}$. The FS detector converts photons into electrical pulses with $\sim 2 \mathrm{~ns}$ width, which are counted by the electronics during a Gate Time Unit (GTU) of $2.5 \mu \mathrm{s}$.

The imaging part of the telescope is an extremely fast, highly pixelized, largeaperture, and wide-FoV digital camera. It is sensitive to near UltraViolet (UV) wavelength band between about 300 and $430 \mathrm{~nm}$ with single photon counting capability. The telescope records the spatial and temporal profile of the UV light emitted as an EAS develops in the atmosphere.

Since the intensity of the observed light depends on the transmittance of the atmosphere, the cloud coverage and the height of the cloud-tops, JEM-EUSO is equipped with an Atmospheric Monitoring (AM) system [22]. To characterize the atmospheric conditions as precisely as possible and thus determine the effective observation aperture with high accuracy, the AM system consists of an InfraRed (IR) camera and a LIDAR (LIght Detection And Ranging) system. Additional information on atmospheric conditions is also extracted from the UV data acquired continuously by the JEM-EUSO telescope itself.

The top panel of Figure 1 illustrates the UHECR observation principle in the JEM-EUSO mission. From an orbit at the altitude of $H_{0} \sim 400 \mathrm{~km}$, the 
Table 1: Parameters of the JEM-EUSO telescope. The values in parenthesis apply at the edge of the FoV, otherwise at the the center of the FoV. The ensquared collection efficiency is the ratio of the number of photons focused within a pixel area to those incident on the entrance aperture of the optics. The ensquared energy is the ratio of photons focused within the area of a pixel to those reaching the FS.

\begin{tabular}{|c|c|c|}
\hline \multicolumn{3}{|l|}{ Parameter } \\
\hline Optics & & \\
\hline Optical aperture & $4.5 \mathrm{~m}^{2}$ & baseline \\
\hline Ensquared collection efficiency & $35 \%(15 \%)$ & for $\lambda=350 \mathrm{~nm}$ \\
\hline Ensquared energy & $86 \%(80 \%)$ & for $\lambda=350 \mathrm{~nm}$ \\
\hline Optical bandwidth & $300-430 \mathrm{~nm}$ & \\
\hline Field of view & $0.85 \mathrm{sr}$ & \\
\hline Observational area & $1.4 \times 10^{5} \mathrm{~km}^{2}$ & for $H_{0}=400 \mathrm{~km}$ \\
\hline$\overline{\text { FS detector and electronics }}$ & & \\
\hline Number of pixels & $3.2 \times 10^{5}$ & \\
\hline Spatial angular resolution & $0.074^{\circ}$ & \\
\hline Pixel size at ground & $0.51 \mathrm{~km}(0.61 \mathrm{~km})$ & for $H_{0}=400 \mathrm{~km}$ \\
\hline Quantum efficiency & $41 \%$ & $\lambda=350 \mathrm{~nm}$ \\
\hline Collection efficiency & $80 \%$ & \\
\hline Cross talk & $<2 \%$ & \\
\hline Transmittance of UV filter & $97 \%$ & for $\lambda=350 \mathrm{~nm}$ \\
\hline Sampling time & $2.5 \mu \mathrm{s}$ & \\
\hline
\end{tabular}




\section{JEM-EUSO Orbit altitude:}
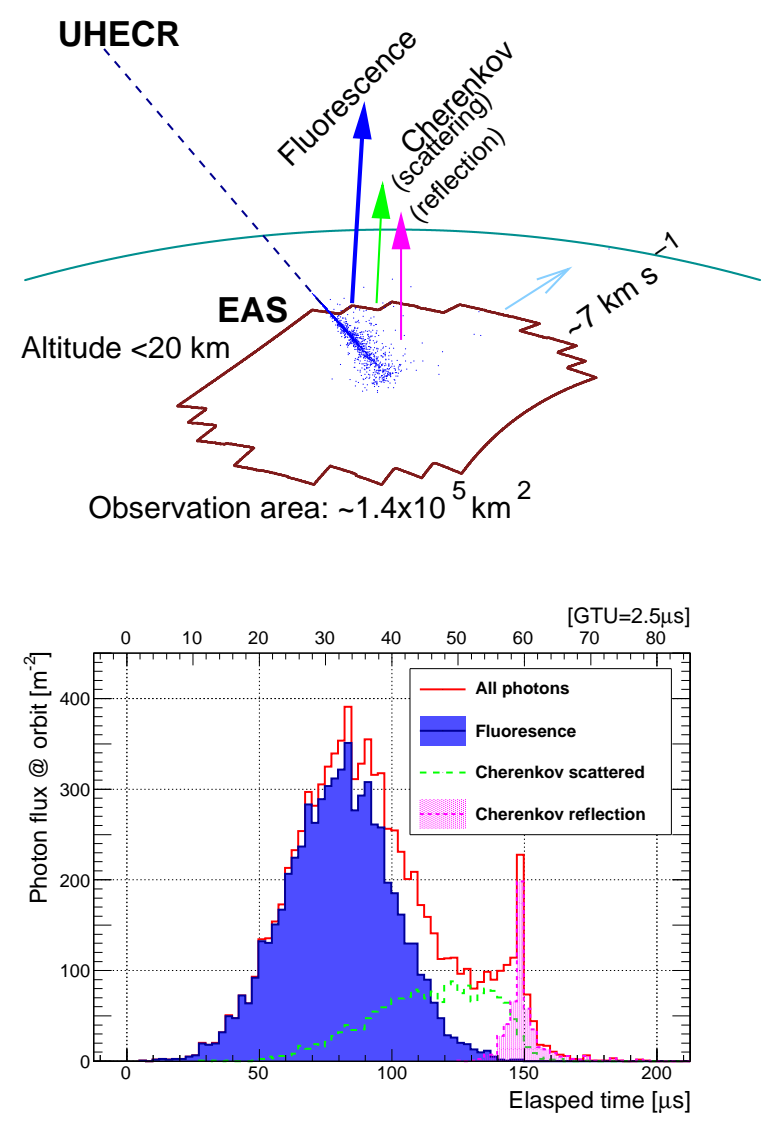

Figure 1: Top: Illustration of UHECR observation principle in the JEM-EUSO mission. For the telescope at $H_{0} \sim 400 \mathrm{~km}$ altitude, the main signals are fluorescence photons along the EAS track and Cherenkov photons diffusely reflected from the Earth's surface. Bottom: Components of the photon signal at the entrance aperture for a standard EAS with $E=$ $10^{20} \mathrm{eV}$ and $\theta=60^{\circ}$ as simulated by ESAF (see Section [5).

JEM-EUSO telescope detects fluorescence and Cherenkov light from EAS. The fluorescence light is emitted isotropically along the EAS track and is observed directly. Since the Cherenkov light is forward-beamed, it is observed either because of scattering in the atmosphere or because of diffuse reflection from the surface of the Earth or a cloud-top. The latter is referred to as 'Cherenkov mark' and it provides additional information about the shower geometry. A $10^{20} \mathrm{eV}$ UHECR produces an EAS with $\mathcal{O}(11)$ particles in the region where the shower reaches its maximum size. Secondary charged particles, predominantly elec- 
trons, excite atmospheric nitrogen molecules that cause UV fluorescence light emitted at characteristic lines in the band $\lambda \sim 300-430 \mathrm{~nm}$. The fluorescence yield has been intensively studied by many groups and is found to be $\sim 3-5$ photons $\mathrm{m}^{-1}$ per electron [23, 24]. During the development of a $10^{20} \mathrm{eV}$ EAS, an order of $10^{15}$ photons are emitted. Seen from $\sim 400 \mathrm{~km}$ distance, the solid angle subtended by a telescope with a few- $\mathrm{m}^{2}$ aperture is $\sim 10^{-11}$ sr. This implies several thousands of photons reach the entrance aperture of the telescope under clear atmospheric conditions.

The arrival time distribution of photons at the entrance aperture, is presented in the bottom panel of Figure 1 The fluorescence light is the dominant component, with smaller contributions coming from reflected and back-scattered Cherenkov light. Since fluorescence light dominates the signal, the energy can be determined with only small corrections for the Cherenkov component. From $H_{0} \sim 400 \mathrm{~km}$, the brightest part of the EAS development, which occurs below $\sim 20 \mathrm{~km}$ altitude, appears always at an almost constant distance, for a fixed location of the EAS in the FoV, regardless of the direction of the EAS, strongly reducing the proximity effects. These are advantageous characteristics of spacebased experiments. In a sense, JEM-EUSO functions as a huge time projection chamber. In addition, Cherenkov light reflected from surface of the ground or cloud-top is useful for providing a time mark for the terminus of the shower.

The orbit of the ISS has an inclination $51.6^{\circ}$ and $H_{0}$ can range between $278 \mathrm{~km}$ and $460 \mathrm{~km}$ according to the operational limits [25]. The sub-satellite speed of ISS and the orbital period are $\sim 7 \mathrm{~km} \mathrm{~s}^{-1}$ and $\sim 90 \mathrm{~min}$, respectively. Apart from effects of orbital decay and operational boost-up, the ISS orbit is approximately circular. $H_{0}$ varies on long-time scale. In the present work, we assume $H_{0}=400 \mathrm{~km}$ as a constant value.

The ISS attitude is normally $+\mathrm{XV} \mathrm{V}+\mathrm{ZLV}$ attitude [26] and deviates from it only for very short periods. $+\mathrm{XV} \mathrm{V}+\mathrm{ZLV}$ is the operational attitude for JEM-EUSO. The JEM-EUSO telescope is designed to point to nadir, referred to as 'nadir mode', as well as to tilt astern to the direction of the motion, referred to as 'tilt mode'. In the following argument, we focus on the case of nadir observation.

The observation area of the Earth's surface is essentially determined by the projection of the FoV of the optics and the area of the FS. The FoV of the optics is estimated using ray tracing simulations [18, 27]. Ray tracing can be used to map the focal surface onto the surface of the Earth as shown in Figure 2 .

Figure 2 shows the outline of the focal surface mapped onto the surface of the Earth (solid curves) and the maps of individual PDMs onto the Earth's surface (dashed curves) for the case when the ISS is located at $H_{0}=400 \mathrm{~km}$. The background in the figure represents the annual average intensity of light pollution measured by the DMSP satellite (see the next section for further details).

The dimensions of the FoV are $\sim 64^{\circ}$ and $\sim 45^{\circ}$ on the major and minor axes, respectively. For these axes, the projected lengths on Earth's surface are $\sim 500 \mathrm{~km}$ and $\sim 330 \mathrm{~km}$, respectively for $H_{0}=400 \mathrm{~km}$. The effective solid angle $\Omega_{\mathrm{FoV}}$ is $\sim 0.85 \mathrm{sr}$. For the planned layout of PDMs on the FS, the size of 


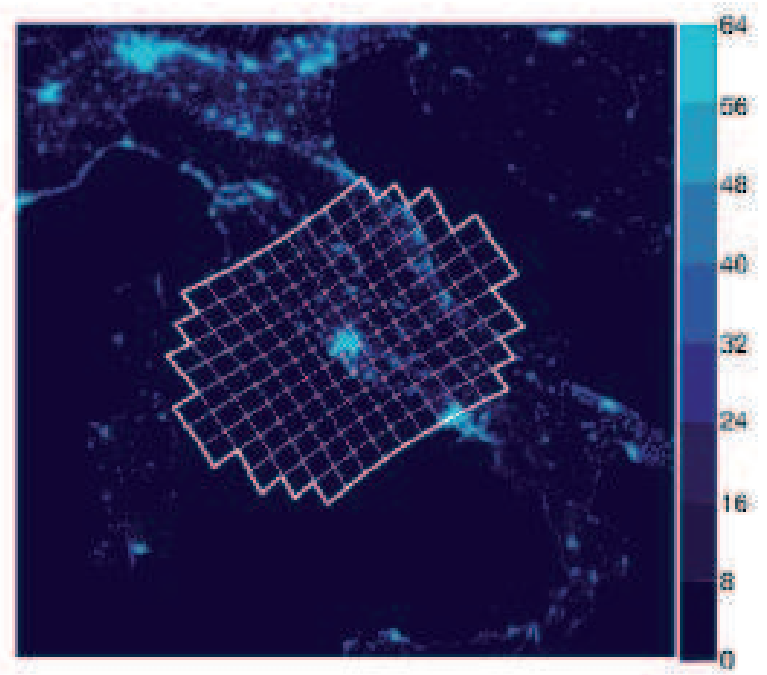

Figure 2: Observation area of JEM-EUSO telescope flying over central Italy. The background in the map shows visible light distribution obtained by DMSP data. The scales denote the values in DMSP units (see Section 3 for details).

the observation area $S_{\text {obs }}$ is a function of $H_{0}$ expressed by:

$$
\begin{aligned}
S_{\mathrm{obs}}\left[\mathrm{km}^{2}\right] & \equiv \Omega_{\mathrm{FoV}} H_{0}{ }^{2} \\
& =1.4 \times 10^{5} \cdot\left(\frac{H_{0}}{400[\mathrm{~km}]}\right)^{2} .
\end{aligned}
$$

It is worth noting that the wide FoV allows measurement of the entire EAS development from the early stage until it fades out or impacts the Earth. This is especially important for EASs from large zenith angles and gamma ray or neutrino induced EASs [28].

\section{Background and observation efficiency}

The UV tracks of an EAS must be discriminated from the UV background. One parameter essential to estimate the JEM-EUSO exposure is the fraction of time during which EAS observation is not hampered by the brightness of the atmosphere. We denote the main component of the intensity of diffuse background light at the JEM-EUSO telescope, $I_{\mathrm{BG}}$, a quantity which is variable over time. We define the observational duty cycle, $\eta$, the fraction of time during 
which the background intensity is lower than a given value $I_{\mathrm{BG}}^{\mathrm{thr}}$. We have:

$$
\eta\left(<I_{\mathrm{BG}}^{\mathrm{thr}}\right)=\eta_{\text {night }} \int_{0}^{I_{\mathrm{BG}}^{\mathrm{thr}}} p\left(I_{\mathrm{BG}}\right) d I_{\mathrm{BG}},
$$

where $\eta_{\text {night }}$ is the nighttime fraction and $p\left(I_{\mathrm{BG}}\right)$ is the probability density function of $I_{\mathrm{BG}}$ over the nighttime defined as the absence of Sun in the visible sky at the orbit level. This requires the zenith angle of the Sun to be greater than $109^{\circ}$ for $H_{0}=400 \mathrm{~km}$ and results in $\eta_{\text {night }}=34 \%$.

Different sources are responsible for lighting the atmosphere in the JEMEUSO FoV, including terrestrial sources like night-glow, TLEs, and local light such as city lights, as well as, extraterrestrial light scattered in the atmosphere, such as moonlight, zodiacal light, and integrated star light. While most of these sources affect the entire FoV, local light only affect portions of the FoV. Therefore, the contribution from local light will be considered separately as a term that decreases the instantaneous aperture of the apparatus.

Moonlight is the largest background component. We estimate moonlight contamination from the phase of the Moon together with its apparent position as seen from the ISS. In our approach, the ISS trajectory provided by NASA SSCweb [29] is traced with 1-min time steps and the moonlight at the top of the atmosphere is estimated according to [30]. For every position of the ISS in the period from 2005 till 2007, the zenith angle of the Sun, and that of the Moon, $\theta_{\mathrm{M}}$, as well as the Moon phase angle, $\beta_{\mathrm{M}}$, are calculated. Background level from reflected moonlight, $I_{\mathrm{M}}$, is evaluated using a modified version of the technique described in 30]. The UV flux from the full Moon is estimated according to the magnitudes and color index from 31]. The apparent visible magnitude at $550 \mathrm{~nm}$ of the full Moon is $V=-12.74$, while the color index $U-V=1.38$. This yields an ultraviolet magnitude of $U=-11.36$ at $360 \mathrm{~nm}$. This corresponds to $2.7 \times 10^{5}$ photons $\mathrm{m}^{-2} \mathrm{~ns}^{-1}$ for $\lambda=300-400 \mathrm{~nm}$. The $\beta_{\mathrm{M}}$-dependence of magnitudes is well approximated in 32 .

The mean albedo of the Earth evaluated from direct satellite measurements has a value close to 0.31 33. Note that such measurements do not distinguish the presence of cloud or other effects that may increase the background intensity. Taking into account the wavelength dependence of the reflectivity [34] as well, we set a conservative value of 0.35 for the reflectivity in the range $\lambda=300-$ $400 \mathrm{~nm}$. Assuming that the radiance of moonlight on the top of the atmosphere is diffusely scattered, the overall intensity of the backscattered moonlight is estimated to be:

$$
I_{\mathrm{M}}=\left[1.6 \times 10^{4} \cdot 10^{-0.4 \times\left(1.5 \cdot\left|\beta_{\mathrm{M}}\right|+4.3 \times 10^{-2} \beta_{M}{ }^{4}\right)}\right] \cos \theta_{\mathrm{M}},
$$

where $\beta_{\mathrm{M}}$ is in radians and $I_{\mathrm{M}}$ has units of photons $\mathrm{m}^{-2} \mathrm{~ns}^{-1} \mathrm{sr}^{-1}$. The overall background intensity, $I_{\mathrm{BG}}$, is given by:

$$
I_{\mathrm{BG}}=I_{\mathrm{M}}\left(\theta_{\mathrm{M}}, \beta_{\mathrm{M}}\right)+I_{0},
$$




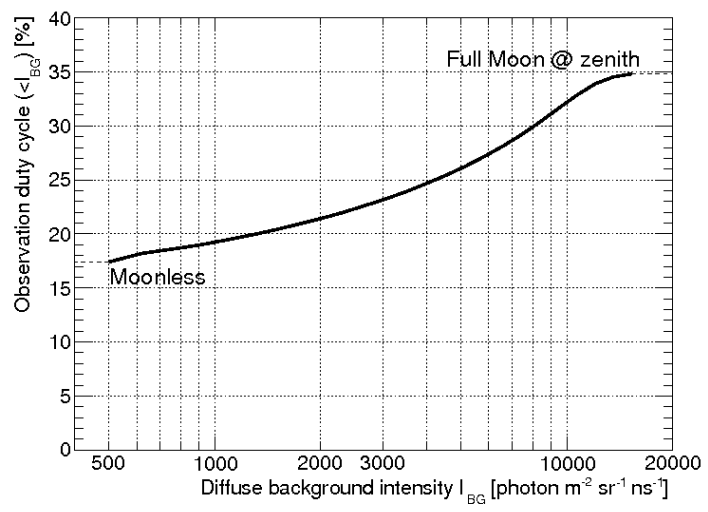

Figure 3: Observational duty cycle $\eta$ as a function of threshold background level $I_{\mathrm{BG}}^{\mathrm{thr}}$.

where $I_{0}$ represents the stable contribution of UV background created mainly by night-glow. In this calculation, $I_{0}$ is assumed to have a constant value of 500 photons $\mathrm{m}^{-2} \mathrm{sr}^{-1} \mathrm{~ns}^{-1}$ in the range $\lambda=300-400 \mathrm{~nm}$ 35, 36, 37]. Taking into account the responses of the optics and FS detectors, the average background level on the MAPMTs corresponds to $\sim 1.1$ photoelectrons $\mathrm{GTU}^{-1}$ per pixel.

Figure 3 shows the observational duty cycle as a function of the accepted background level according to Equation (2). The fraction of time during which $I_{\mathrm{BG}}$ is less than 1500 photons $\mathrm{m}^{-2} \mathrm{sr}^{-1} \mathrm{~ns}^{-1}$ is $20 \%-21 \%$ resulting in an average background level of 550 photons $\mathrm{m}^{-2} \mathrm{sr}^{-1} \mathrm{~ns}^{-1}$ under this condition. The threshold of 1500 photons $\mathrm{m}^{2} \mathrm{~ns}^{-1} \mathrm{sr}^{-1}$ is chosen as a reference. This corresponds to the decrease of signal to noise ratio by a factor of $\sim \sqrt{3}$ in comparison to the case of $I_{\mathrm{BG}}=500 \mathrm{~m}^{2} \mathrm{~ns}^{-1} \mathrm{sr}^{-1}$, which still guarantees EAS observation. The effects of variable background level will be discussed in Section 7 Of course, the operation of the instrument is not limited by this value, so this is a conservative estimate for the highest energies, where measurement can be performed even in a higher background condition. In the following discussion, we assume $\eta_{0} \sim 20 \%$ as the reference value for the observational duty cycle.

In addition to the diffuse sources of background, there are intermittent local sources such as lightening flashes, auroras, or city lights. In the cases of lightening and TLEs, estimates of the reduction in observational duty cycle and instantaneous aperture are performed assuming the rate of events detected by Tatiana satellite [35]. We further assume that the EAS measurement is not possible in all PDMs as long as the location of the event is within the JEM-EUSO FoV. For a conservative estimation, we applied $\sim 70$ s to all events, that corresponds to the maximum time a light source takes to traverse the major axis of the FoV. Even with these extremely conservative assumptions, the overall effect is less than $\sim 2 \%$. Moreover, as lightening is very often associated with high clouds, most of this effect is already included in cloud inefficiency, as described 
Table 2: Fraction of pixels with visible light intensities $\leq 3,>3$ and $>7$ (DMSP units) in spatial resolutions of DMSP (left columns) and of JEM-EUSO PDM (right columns).

\begin{tabular}{c|ccc|ccc}
\hline & \multicolumn{2}{|c|}{ DMSP } & pixel resolution & \multicolumn{3}{c}{ JEM-EUSO PDM resolution } \\
\cline { 2 - 7 } & $\leq 3$ & $>3$ & $>7$ & $\leq 3$ & $>3$ & $>7$ \\
\hline All & $96 \%$ & $4 \%$ & $2 \%$ & $87 \%$ & $13 \%$ & $7 \%$ \\
Land & $85 \%$ & $15 \%$ & $6 \%$ & $58 \%$ & $42 \%$ & $25 \%$ \\
Ocean & $99.8 \%$ & $0.2 \%$ & $0.1 \%$ & $99.1 \%$ & $0.9 \%$ & $0.4 \%$ \\
\hline
\end{tabular}

in the next section.

To estimate the reduction in observational duty cycle and aperture due to the occurrence of auroras, we used the $K_{\mathrm{p}}$ index to describe the geomagnetic activity, as well as, the geomagnetic latitude and longitude of ISS during years 2001 and 2006. These two years were selected as they were close to solar minimum and solar maximum, respectively. In the estimation, it was assumed that no measurement can be performed when the $K_{p}$ index for ISS geomagnetic latitude is equal or higher than Auroral Boundary Index [38]. Even in the case of maximum solar activity the effect is of the level of $\sim 1 \%$.

To evaluate the effect of the stationary light sources on the Earth, which are mainly anthropogenic, we use the Defense Meteorological Satellite Program (DMSP) 39] database. Annual averages of light intensities for cloud-free moonless night are used to estimate the presence of local light along the ISS trajectory. The DMSP data provide the light intensity in 64 different levels on a 30arcsecond grid in latitude and longitude in the wavelength range $350 \mathrm{~nm}-2 \mu \mathrm{m}$. The units are arbitrary, with equally spaced steps. The stationary background is dominated by visible light. As an example, the average level of background around central Italy is shown in Figure 2, As the trigger system in JEM-EUSO works at the PDM level (see Section 5), we discuss the impact of local light at PDM level here.

Table 2 summarizes the results on the visible intensity from DMSP data over the region between $51.6^{\circ} \mathrm{S}$ and $51.6^{\circ} \mathrm{N}$ latitudes which is covered by ISS trajectory. From the DMSP data, which have their own spatial resolution, the average intensity is 2.6 in DMSP units. This value is mainly determined by the background over the ocean which represents $72 \%$ of the JEM-EUSO observational region. In the following, we make the conservative assumption that no measurement of EASs is performed if, in a region viewed by a PDM, there is at least one pixel which detects a light intensity which exceeds the average level by a factor of 3 or more (higher than 7 in a DMSP units). With this assumption, the inefficiency of the instantaneous aperture is of the order of $\sim 7 \%$. It is important to remember that Tatiana measurements 35] - without focusing optics - indicate a 2-3 times higher intensity in UV above big cities such as Mexico City and Houston compared to the average background level over the ocean. Finally, by combining the above estimations for lightnings $(\sim 2 \%)$, auroras $(\sim 1 \%)$ and DMSP data the overall loss of coverage is $f_{\text {loc }} \sim 10 \%$. 


\section{Climatological distribution of clouds}

In the case of space-based observation, reconstructing an EAS event is feasible, even in the presence of clouds, if the EAS maximum is sufficiently above the cloud-top altitude, $H_{\mathrm{C}}$ 7, 40]. In some cases, the presence of specific cloud types may even be an advantage (see Section 6), which is contrary to groundbased observation. An optically thick cloud represents a very uniform layer which enhances the intensity of the Cherenkov mark and gives a brighter end point of the track. Of course, the cloud-top height should be known with reasonable uncertainty $(\sim 0.5-1 \mathrm{~km})$, and for that, the AM system is used. In the case of optically thin clouds or very inclined showers, however, the Cherenkov mark is not well defined. Therefore, it is mandatory to develop alternative reconstruction algorithms which do not rely on the detection of the Cherenkov mark [41]. The combined use of algorithms based on different approaches on an event-by-event basis helps to prevent, or at least tag, misreconstructed events. Thin clouds with optical depths $\tau_{\mathrm{C}}<1$ (typically cirrus) may affect the estimation of the energy, but the arrival direction can be determined with acceptable uncertainty. In such a case, the estimated energy is likely to be lower than the true one, adding to a given reconstructed energy bin an event whose true energy is in fact larger, and thus whose angular deflection is a priori smaller - not the opposite. Even though such a situation may alter the quantitative estimates of the anisotropy as a function of energy, some anisotropy analyses will still be interesting to perform with such events, notably those assessing a lower limit on anisotropy. Optically thick clouds, with $\tau_{\mathrm{C}}>1$, strongly influence the measurement only if they are located at high altitudes. For example, EASs from a $60^{\circ}$ zenith angle and energy $\sim 10^{20} \mathrm{eV}$ reach their maxima at an altitude around $\sim 6.5 \mathrm{~km}$, much higher than the typical range of stratus. The effect of clouds is, therefore, to limit the instantaneous aperture by obscuring portions of the FoV.

In order to quantify the effect of cloud contamination, a study of the climatological distribution of clouds, as a function of cloud-top altitude, optical depth, and geographical location has been performed using the meteorological databases TOVS, ISCCP and CACOLO.

The NASA project TOVS (TIROS Operational Vertical Sounder) [42] on board NOAA's TIROS series of polar orbiting satellites provides data with a good spectral distribution, as well as optical depth and altitude of clouds, which are obtained applying a radiative transport model [42]. In this study, data from 1988 to 1994 have been used, including both land and ocean data.

The International Satellite Cloud Climatology Project (ISCCP) 43] was established in 1982 to collect and analyze satellite radiance measurements needed to infer the global distribution of clouds, their properties and their diurnal, seasonal and inter-annual variations. The ISCCP has developed cloud detection schemes using visible and IR window radiance (IR during nighttime and daytime, and visible during daytime). The data from 1983 to 2008 have been used in this analysis. Data are given on a 2.5-degree grid in latitude and longitude.

The CACOLO (Climatic Atlas of Clouds Over Land and Ocean data) database 


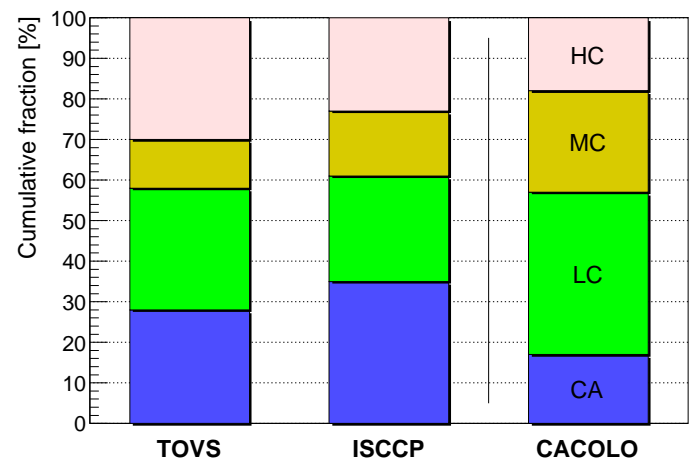

Figure 4: Comparison of the TOVS, ISCCP and CACOLO databases for the relative cloud occurrence in different meteorological situations. The data correspond to daytime, with a weighted average between land and ocean. The classification of the TOVS and ISCCP data is based on the cloud-top altitude while for CACOLO it is based on the cloud-bottom. High clouds are defined by $H_{C}>6.5 \mathrm{~km}$; Middle clouds by $H_{C}=3.2-6.5 \mathrm{~km}$; Low clouds by $H_{C}$ $<3.2 \mathrm{~km}$. The abbreviation are defined as HC: High Clouds; MC: Middle Clouds, LC: Low Clouds and CA: Clear Atmosphere.

[44] presents maps introduced in the atlases of cloud climatological data obtained from visual observations from Earth. Most data are given at a 5-degree resolution in latitude and longitude. The land data are based on analysis of visual cloud observations performed at weather stations on continents and islands over a 26-year period (1971-1996). The ocean maps are based on analysis of cloud observations made from ships over a 44-year period (1954-1997).

Systematic differences between these databases have been evaluated. As previously explained, the ISCCP and CACOLO data divide the clouds only in low $\left(H_{\mathrm{C}}<3.2 \mathrm{~km}\right)$, middle $\left(H_{\mathrm{C}}=3.2-6.5 \mathrm{~km}\right)$ and high types $\left(H_{\mathrm{C}}>6.5 \mathrm{~km}\right)$ without distinguishing according to their optical depths. Some care has to be taken with CACOLO results, as these data are based on observations from the ground, so the cloud altitude refers to the cloud bottom. Since the CACOLO data characterize well the cloud occurrence in the lower part of the atmosphere, they nicely complement the observations from space.

Figure 4 shows a comparison of cloud distribution in the troposphere among the three datasets where land and ocean data are combined in a weighted average. Only daytime data are shown, since CACOLO employs visual observation.

Despite the fact that significant differences exist among the three databases for each of the 4 categories shown, if one considers the case of good JEM-EUSO conditions, namely low clouds or clear atmosphere, the three datasets are in reasonable agreement, with a minimum of $57 \%$ for CACOLO to a maximum of $61 \%$ in case of ISCCP. The difference among high cloud measurements might be due to the fact that CACOLO data are taken by ship and weather stations only in the visual band. This could result in a smaller fraction of high clouds, 
Table 3: Relative occurrence of clouds over the ISS orbit, taken from the TOVS database for nighttime, are presented as a matrix of cloud-top altitude vs optical depth for all location and only ocean.

\begin{tabular}{|c|c|c|c|c|}
\hline \multirow{2}{*}{$\begin{array}{l}\text { Cloud-top } \\
\text { altitude } H_{\mathrm{C}}\end{array}$} & \multicolumn{4}{|c|}{ Optical depth $\tau_{\mathrm{C}}$} \\
\hline & $<0.1$ & $0.1-1$ & $1-2$ & $>2$ \\
\hline & \multicolumn{4}{|c|}{ All data } \\
\hline$>10 \mathrm{~km}$ & $1.2 \%$ & $5.0 \%$ & $2.5 \%$ & $5.0 \%$ \\
\hline $6.5-10 \mathrm{~km}$ & $<0.1 \%$ & $3.2 \%$ & $4.2 \%$ & $8.5 \%$ \\
\hline $3.2-6.5 \mathrm{~km}$ & $<0.1 \%$ & $2.0 \%$ & $3.0 \%$ & $6.0 \%$ \\
\hline$<3.2 \mathrm{~km}$ & $31 \%$ & $6.4 \%$ & $6.0 \%$ & $16 \%$ \\
\hline & \multicolumn{4}{|c|}{ Ocean data } \\
\hline$>10 \mathrm{~km}$ & $0.1 \%$ & $5.0 \%$ & $2.4 \%$ & $4.7 \%$ \\
\hline $6.5-10 \mathrm{~km}$ & $0.1 \%$ & $3.2 \%$ & $4.3 \%$ & $9.2 \%$ \\
\hline $3.2-6.5 \mathrm{~km}$ & $0.1 \%$ & $2.1 \%$ & $3.1 \%$ & $5.7 \%$ \\
\hline$<3.2 \mathrm{~km}$ & $29 \%$ & $6.6 \%$ & $6.5 \%$ & $17 \%$ \\
\hline
\end{tabular}

especially in presence of low and middle altitude clouds. In contrast, TOVS data are taken by satellites, therefore, low and middle altitude clouds may be underestimated due to the obscuration by high clouds. ISCCP data show a more uniform cloud occurrence among the different atmospheric levels most probably because they are taken from satellite in the visual and IR bands that enables distinguishing the various levels. Since the TOVS data show the highest fraction of high clouds, which are the most critical in case of EAS observation from space, estimates of the fraction of EASs measurable by JEM-EUSO using Table 3 can be considered as conservative.

Table 3 reports TOVS data on the occurrence of each cloud category during nighttime on the globe and above the ocean only. The results apply only to the region of the ISS trajectory and account for the residence time of the ISS as a function of latitude.

A comparison between day and night cloud coverage has been performed for clouds above land as higher variations are expected in comparison with the daynight variation above the ocean. Slight differences among tables exist (typically $\sim 5 \%$ ), though the general trend seems to be independent of the geographical and temporal conditions [45]. In any case, only nighttime conditions are relevant for JEM-EUSO. By comparing TOVS results in Figure 4 and Table 3 , no significant difference seems to exist between the high (30\% also in Table 3) and middle (11\% in Table 3) cloud occurrence in daytime and nighttime. The clear atmosphere seems to be slightly more frequent in nighttime compared to daytime.

Finally, the ISCCP data have been used to check the dependence of the above results on latitude. Results are summarized in Figure [5]

In general the occurrence of low clouds and clear atmosphere is slightly higher over the oceans. It is important to remember that ocean accounts for $72 \%$ of the time for the ISS orbit. High clouds are particularly frequent in the equatorial 

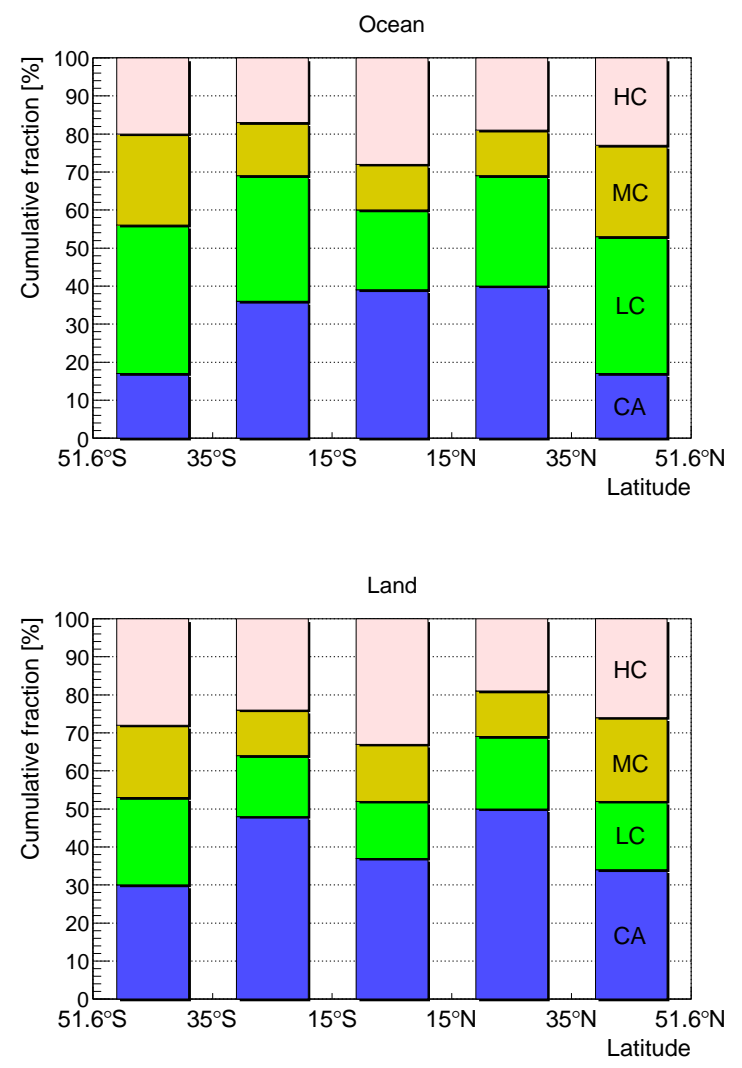

Figure 5: Distributions of the cloud properties over the ocean (top panel) and the land (bottom) are given for 5 latitudinal zones, using ISCCP data. The abbreviation are defined as HC: High Clouds; MC: Middle Clouds, LC: Low Clouds and CA: Clear Atmosphere. Data refer to daytime.

region. This is expected, due to the presence of the cloudiness associated to the Inter Tropical Convergence Zone.

\section{Trigger scheme and shower simulation}

Another key parameter in determining the exposure is the EAS trigger efficiency of the instrument. This is strongly dependent on the characteristics of the optics and FS detector, as well as the darkness of the atmosphere. Therefore, the trigger logic explained in the following was defined as a trade-off between the available power and telemetry budgets of the instrument, the response of the detector, as explained later in this section, and the necessity of coping with background fluctuations whose excess at tenths microsecond level on an MAPMT basis could mimic the presence of a signal from an EAS. 
To reject the background, the JEM-EUSO electronics employ two trigger levels. The trigger scheme relies on the partitioning of the FS onto PDMs, which are large enough to contain a substantial part of the imaged trace under investigation, as explained below.

The $1^{\text {st }}$ trigger level rejects most of the background fluctuations by requiring a locally persistent signal above average background lasting a few GTUs. In this trigger level, referred to as Persistent Track Trigger (PPT), pixels are grouped in $3 \times 3$ boxes. A trigger is issued if for a certain number of consecutive GTUs, $N_{\text {pst }}$, there is at least one pixel in the box with an activity equal to or higher than a preset threshold, $n_{\mathrm{thr}}^{\mathrm{pix}}$, and the total number of detected photoelectrons in the box is higher than a preset value $n_{\mathrm{thr}}^{\mathrm{box}}$. $N_{\text {pst }}$ is set to 5 GTUs in the current simulations, while $n_{\mathrm{thr}}^{\mathrm{pix}}$ and $n_{\mathrm{thr}}^{\mathrm{box}}$ are set as a function of $I_{\mathrm{BG}}$ in order to keep the rate of triggers on fake events at few $\mathrm{Hz}$ per PDM. For an average background level of 1.1 photoelectron $\mathrm{GTU}^{-1}$ per pixel, $n_{\mathrm{thr}}^{\mathrm{pix}}$ is set to 2 and $n_{\mathrm{thr}}^{\mathrm{box}}$ to 32 .

The $2^{\text {nd }}$ trigger level [46], referred to as the Linear Track Trigger on Cluster Control Board (CCB_LTT), follows the movement of the EAS spot inside the PDM over a predefined time window to distinguish the unique pattern of an EAS from the background. Starting from the location where the PPT trigger is issued, the CCB_LTT trigger algorithm defines a box of $3 \times 3$ pixels around this trigger seed, then moves the box every GTU. The box is moved along predefined lines to search for the direction of the EAS. The photon count along each line is integrated each GTU by summing up the counts $n^{\text {pix }}$ of the pixels in the box that in such GTU satisfy the condition $n^{\text {pix }} \geq n_{\text {thr }}^{\text {pix }}$. The integration is performed for 15 consecutive GTUs. If the integration along a direction exceeds a prefixed threshold, eg. 97 counts under this background level, the CCB_LTT trigger is issued. In order to follow the movement of the spot on the focal surface, the speed and the direction in terms of detector pixels is calculated according to:

$$
\begin{aligned}
\hat{\theta} & =2 \arctan \left(\frac{\Delta L}{c \cdot \Delta t} \cdot \sqrt{\Delta x^{2}+\Delta y^{2}}\right) \\
\hat{\varphi} & =\arctan \left(\frac{\Delta y}{\Delta x}\right)
\end{aligned}
$$

where $\hat{\theta}$ and $\hat{\varphi}$ are, respectively, the polar angle and azimuthal angle in a spherical coordinate system whose polar axis is aligned along the line of sight of the pixel, $c$ is the speed of light, $\Delta x$ and $\Delta y$ are the number of pixels crossed in a time $\Delta t$, and $\Delta L$ is the projected length of the pixel FoV on the Earth's surface, which is given in Table 1 .

Since the incoming direction of the EAS is unknown, the CCB_LTT trigger tries directions which fully cover the phase space $\left(\hat{\theta}=5^{\circ}, 10^{\circ}, \ldots, 85^{\circ}\right.$ and $\hat{\varphi}=$ $\left.5^{\circ}, 10^{\circ}, \ldots, 355^{\circ}\right)$, which means that the directions in which the box should move are defined before starting the integration for 15 GTUs. The integrated count value will have a maximum when the nearest direction to the correct one is selected because in this case the integrating box will most closely follow the 
EAS track. The $I_{\mathrm{BG}}$-dependent threshold on the total number of counts inside the track is tuned to reduce the fake events to a rate of $0.1 \mathrm{~Hz}$ on the entire FS. The two trigger levels combined operate a reduction in rate by $\sim 2 \times 10^{-7}$ at PDM level. When a trigger is issued, a sufficiently large part of the FoV (a few PDMs) is acquired in order to image the region around the EAS track.

The trigger rate for real EASs is less than $\sim 1 \%$ of the total trigger rate, depending on the background intensity. A more comprehensive review of the trigger scheme is given in [47]. Besides the fact that the threshold in energy is affected by the darkness of the atmosphere and the photon collecting power of the telescope, it is important to underline here that the trigger system on a space-based detector has to be much more selective than a ground-based experiment because of telemetry constraints. This limits the threshold in energy. On the other side, the fraction of scientific data in the sample will be of high quality. Thus, it is expected that further quality cuts applied in the offline analysis will not cause a significant reduction of data.

In order to evaluate the detector response to the EAS observation, we use the Euso Simulation and Analysis Framework (ESAF). A detailed description of the software can be found in [48]. In the following analysis, the Greisen-Ilina-Linsley (GIL) function [49] is used as parametric generator to reproduce the profile as a function of slant depth. The GIL function is optimized to reproduce EAS from hadronic particles simulated by CORSIKA [50] with the QGSJET01 hadronic interaction model [51]. Proton showers have been simulated for the analyses presented in this paper. This is motivated by the fact that they develop deeper in the atmosphere, which results in a higher atmospheric absorption and higher cloud impact 2. Therefore, the results that are discussed in the following sections constitute a conservative estimation on the performance of the instrument.

In the present work, the fluorescence yield, which constitutes one of the larger uncertainties in energy determination, is taken from [52]. In the atmosphere, UV photon propagation is strongly affected by Rayleigh scattering and absorption by ozone for wavelengths $\lesssim 320 \mathrm{~nm}$. These processes along with the atmospheric profile are modeled with the LOWTRAN package [53].

The detector simulation includes optical ray tracing, PDM layout on the FS, UV filter, MAPMT performance and trigger algorithm. For the optics response, the simulation code described in [18] has been adopted in the present analysis. A parametrization of the MAPMT is included in the electronics simulation. All the effects like quantum efficiency, including the dependence on photon inclination, collection efficiency and cross talk are also taken into account pixel by pixel within one MAPMT as summarized in Table 1

\footnotetext{
${ }^{2}$ The EAS observation from space has a better visibility of the early stages of the shower development compared to ground-based observation. Iron showers tend to cascade higher in atmosphere compared to proton ones and the apparent length of the EAS before impacting on the Earth's surface or on a cloud top is a bit longer. Simulation results indicate in case of iron showers that a slightly higher number of photons reaches JEM-EUSO in comparison to proton showers with same energy and geometry. This results in a slightly improved trigger efficiency and increased overall exposure in case of iron showers.
} 


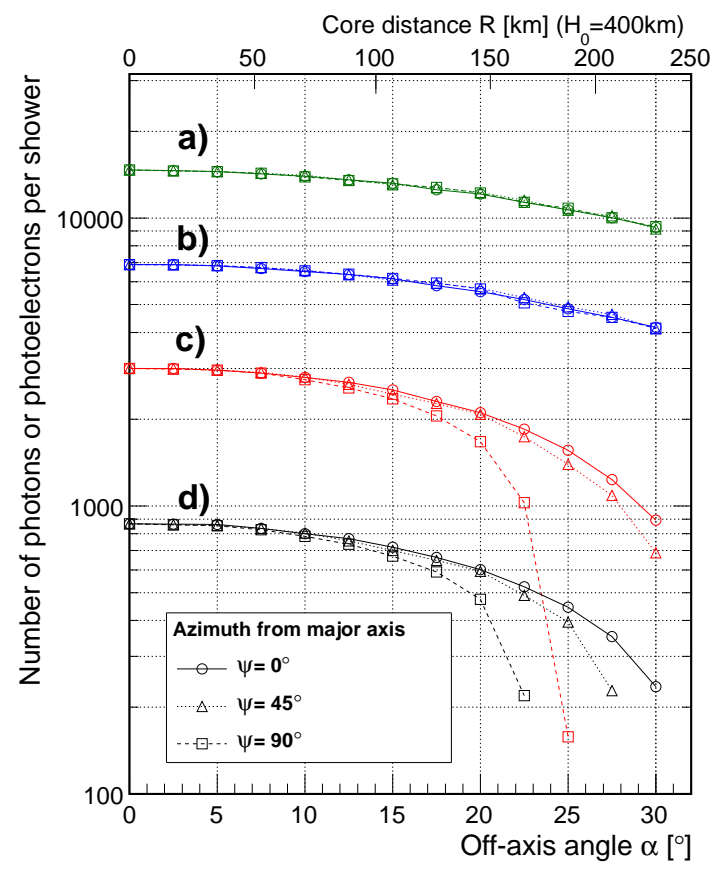

Figure 6: Number of photons and photoelectrons as a function of off-axis angle $\alpha$ of the core location from nadir, obtained by simulating 100 EASs with $10^{20} \mathrm{eV}$ and $\theta=60^{\circ}$. The off-axis angle is the angle between the core location and the nadir axis. To demonstrate the azimuthal dependence of the optics, three different directions are shown by circles $\left(\psi=0^{\circ}\right)$, triangles $\left(\psi=45^{\circ}\right)$ and squares $\left(\psi=90^{\circ}\right)$ where $\psi$ is the angle from the major axis of the optics. The scale on top is the radial distance from the center of FoV on the Earth's surface. In the figure, the different stages are compared: a) photons from the shower axis directed toward the JEM-EUSO entrance aperture; b) photons reaching JEM-EUSO entrance aperture; c) photons reaching the FS and d) detected signal (photoelectrons).

Figure 6 shows the number of photons and signals for the average of 100 EASs with $E=10^{20} \mathrm{eV}$ and $\theta=60^{\circ}$. To demonstrate the radial and azimuthal dependent response of the detector, mostly due to optical vignetting, we simulate EAS with different core locations. The horizontal axis shows the off-axis angle $\alpha$ of the core location with respect to the optical axis corresponding to the direction of nadir. The scale on the top indicates the core distance $R$ from the center of FOV $\left(\sim H_{0} \tan \alpha\right)$. The detector design is symmetric in each quadrant. The azimuthal dependence on the optics is, therefore, only tested for the case $\psi=0^{\circ}, \psi=45^{\circ}$ and $\psi=90^{\circ}$, where $\psi$ is the azimuthal angle of the focusing position from the major axis of the FS.

Independent of $\psi$, the numbers of photons at the entrance aperture, namely stages a) and b) in Figure 6, depend only on the distance between the telescope and EAS, and on an entrance aperture of a given solid angle, so they are 
roughly proportional to $\cos \alpha$. We recall here, as explained in Section 2 that the proximity effects are negligible for a space-based observation. The ratio of b) to a) corresponds to the average transmittance for photons reaching JEM-EUSO from the position where they are produced either by emission or scattering. As the Cherenkov light has continuous spectrum, we simulated the wavelength range up to $485 \mathrm{~nm}$ where the photon detection efficiency is negligible. Over the FoV of JEM-EUSO telescope ( $\alpha \lesssim 30^{\circ}$, or half of FoV for the major axis), the variation of the number of photons at the entrance aperture is within $\sim 1.7$.

The decrease from b) to c) indicates effects in the optics, such as the absorption and scattering of photons in lenses, characteristic aberration and obscuration by the support structure.

The ratio of d) to c) reflects the efficiency of the FS detector and is generally determined by the detection efficiency (product of collection efficiency and quantum efficiency) of MAPMTs and transmittance of UV filter (Schott BG3 filter [54]). Photons may be lost in part when they are focused on void areas such as gaps among PDMs. For $\psi=0^{\circ}$ the difference between the center and edge of the FoV is a factor of $\sim 2$. A significant dependence on $\psi$ emerges at $\alpha \sim 15^{\circ}$. This is because some of the photons arriving from angles close to the minor axis $\left(\psi=90^{\circ}\right)$ are bent to the internal lenses on segments that have been removed in side-cut optics. For $\alpha \gtrsim 23^{\circ}$, no PDM is present on the FS along the minor axis of the optics. Note, however, that this effect only appears between $\psi \sim 45^{\circ}$ and $\psi \sim 90^{\circ}$ and the range of corresponding angles in the quadrants where the segment crosses a circular part of the lens.

The signal is then amplified using a parametrization of the measured gain and the resulting output current is collected and treated by the Front End Electronics. A threshold is set on the MAPMT output current in order to accept or reject the signal count.

The trigger architecture and the parameters used in PTT and CCB_LTT trigger algorithms have been optimized using ESAF and stand alone Monte Carlo simulations to reduce the fake trigger rate from background fluctuations to an acceptable level, exploiting the detector response such as ensquared energy of the optics, and detection efficiency, cross-talk among pixels, etc.

The ESAF code reconstructs the EAS energy, arrival direction, and longitudinal development of simulated events, and it is used to check the accuracy of reconstruction. This also provides feedback that is useful for the development of analytical algorithms and hardware to improve the performance of the detector.

In Figure 7 top panel shows the projected tracks on the Earth's surface for EASs with $E=10^{20} \mathrm{eV}$ and zenith angles of a) $\theta=30^{\circ}$, b) $\theta=60^{\circ}$ and c) $\theta=75^{\circ}$ along with the map for the entire FoV in the sub-panel. Bottom panel shows the image on the FS for the case b) in which the integrated counts for each pixel are indicated. The regions enclosed by thick dashed lines in both panels refer to the same PDM.

Figure 8 shows the arrival time distribution of photons at the telescope entrance aperture from the EASs shown in Figure 7 The shaded histogram is for $\theta=60^{\circ}$ and those with solid and dashed lines are for $\theta=75^{\circ}$ and $\theta=30^{\circ}$, respectively. 

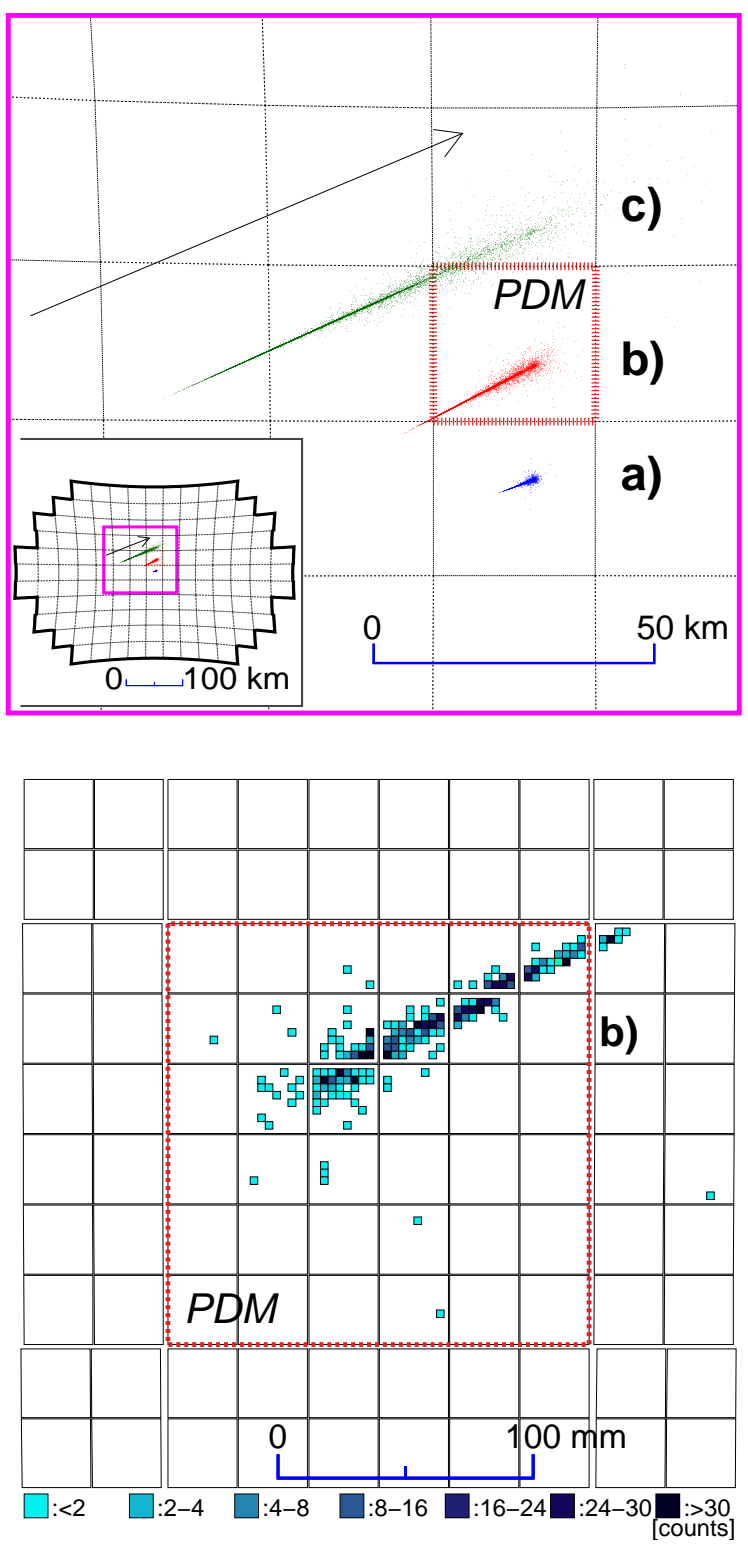

Figure 7: Top panel shows the projected tracks on the Earth's surface for EASs with $E=$ $10^{20} \mathrm{eV}$ and zenith angles of a) $\theta=30^{\circ}$, b) $\theta=60^{\circ}$ and c) $\theta=75^{\circ}$. The dashed curves indicate the corresponding areas for the FoV of individual PDMs. In the sub-panel, the corresponding area of the plot is represented by solid lines within the entire FoV. Bottom panel shows the image on the FS for the case b). The large squares denotes PMTs. The matrix of pixels are indicated with the integrate counts in discrete scale. The regions enclosed by thick dashed lines in both panels refer to the same PDM. 


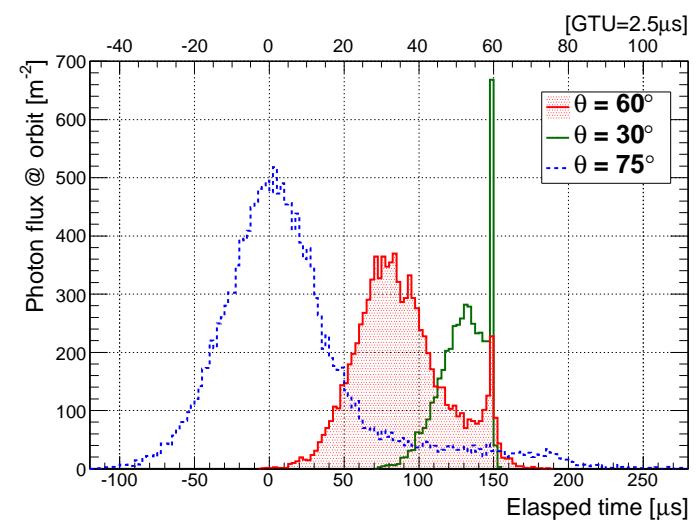

Figure 8: Arrival time distribution of photons at the telescope entrance aperture from the same EASs shown in Figure 7 Shaded histogram denotes the case of $\theta=60^{\circ}$ and those with solid and dashed lines are for the cases of $\theta=75^{\circ}$ and $\theta=30^{\circ}$, respectively.

Up to zenith angles $\theta \sim 60^{\circ}$, the EAS is fully contained in an FoV equivalent to that of one PDM. It reaches two PDMs around $\theta \sim 75^{\circ}$. This is the reason the trigger architecture is based on the PDM scale. The typical FoV of a PDM for $H_{0}=400 \mathrm{~km}$ is about $30 \mathrm{~km}$ on a side $\left(\sim 1000 \mathrm{~km}^{2}\right)$. This means that the entire FS can be considered as the sum of 137 quasi-independent sub-detectors corresponding to PDMs. This is important for evaluating the effects of clouds and city lights. It should be mentioned here that when a trigger is issued on a PDM, the data of the neighboring PDMs are also retrieved. Another important consideration is that more inclined EASs will give higher signals, either at EAS maximum or as total integrated light. This can be used to extend the energy range of measurement to lower energies by simple geometrical cuts. Moreover, inclined showers will allow almost fully calorimetric measurement of the EAS because the entire profile will be visible. This is generally not the case of ground-based detectors, which typically view up to $60^{\circ}$, and for which the EAS is truncated at ground in many cases.

In conclusion, the three main players which define the trigger efficiency for a specific night-glow background level and atmospheric conditions are the optics response (most relevant), the zenith angle of the EAS (a factor of 2-3 in the collected light between quite inclined and vertical showers), and the distance effect ( $\sim 25 \%$ difference in the total number of photons reaching the pupil from the same EAS located at the center or at the edge of the FoV). Each of these three effects can easily be identified by means of simple geometrical cuts on the zenith angle of the EAS and/or on its core location and accounted for given the intrinsic characteristics of the detector. As an example, this means that the evolution of the exposure without geometrical cuts as a function of energy around the threshold can be verified by applying straightforward cuts on selected sub-samples of data where the aperture is known to be flat. This guarantees 


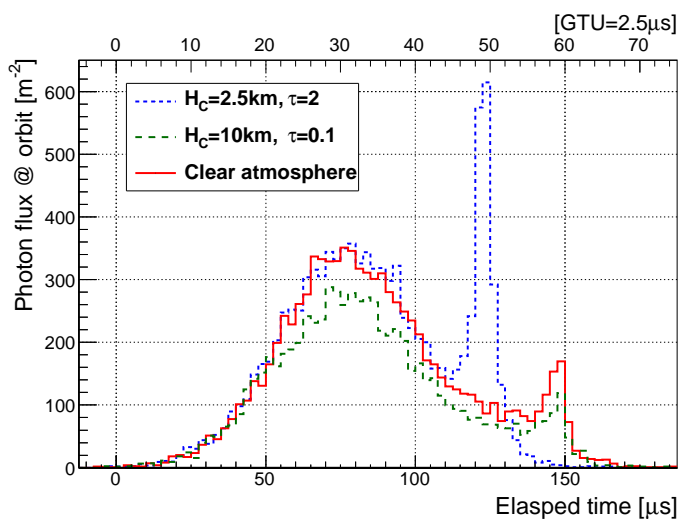

Figure 9: Arrival time distribution of photons to the telescope per $\mathrm{m}^{2}$ from an EAS of $E=$ $10^{20} \mathrm{eV}$ and $\theta=60^{\circ}$ with different cloud conditions. Dashed and dotted lines correspond to the cases of cirrus- and stratus-like test clouds along with solid line for the clear atmosphere case.

the quality of the data even if the aperture without geometrical cuts has not reached the plateau yet.

To include clouds in ESAF, a uniform and homogeneous layer is assumed. Physical parameters considered for the cloud are obtained from a 'test cloud' layer defined by three input parameters: a) optical depth $\tau_{\mathrm{C}}$, b) altitude $H_{\mathrm{C}}$, that yields a transmittance, $\exp \left(-\tau_{\mathrm{C}}\right)$ and c) physical thickness.

In Figure 9 arrival time distribution of photons to the telescope of typical EAS events with zenith angle of $60^{\circ}$ are shown for cirrus- $\left(H_{\mathrm{C}}=10 \mathrm{~km}\right.$ and $\tau_{\mathrm{C}}=$ $0.1)$ and stratus- like $\left(H_{\mathrm{C}}=2.5 \mathrm{~km}\right.$ and $\left.\tau_{\mathrm{C}}=2\right)$ test clouds. For comparison, the arrival time distribution of photons in clear atmosphere of the same EAS is shown.

In case of a cirrus-like cloud at high altitudes, the signals from EAS are attenuated according to the optical depth, while the EAS image and its time evolution allow determination of the arrival direction. The reflected signals of Cherenkov light from the landing surface are also observed.

As mentioned in the previous section, for stratus-like clouds with large $\tau_{\mathrm{C}}$ at lower altitudes, most of the signal from EAS is observed without attenuation when the cloud is well below the altitude of the shower maximum. Such clouds also produce very intense reflected Cherenkov signals even larger than in the clear atmosphere case. This may enhance the capability of triggering particular types of EAS such as low zenith angle events. Moreover, the reconstruction of the EAS geometry may benefit from such high reflectivity since the location of the impact on the cloud is more accurately determined. On the other hand, a dedicated algorithm will be needed to disentangle the contribution of Cherenkov light from fluorescence light in estimating the energy of the event. 


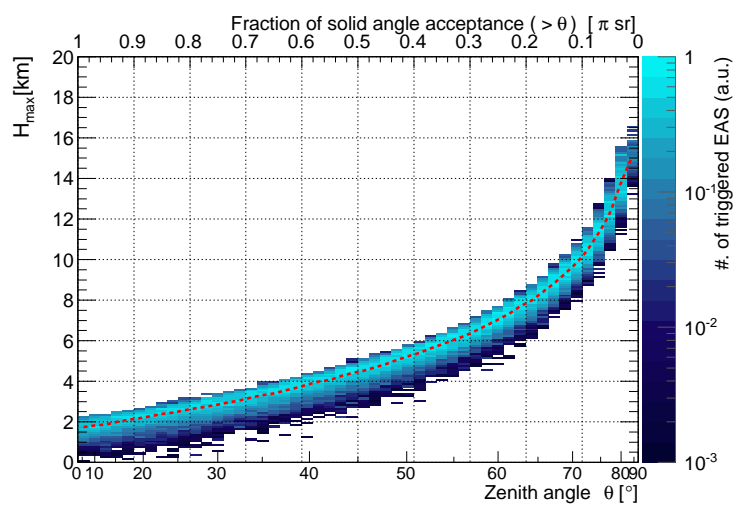

Figure 10: Geometrical aperture as a function of energy. The filled circles and squares indicate geometrical apertures for the entire observation area and $R<150 \mathrm{~km}$ respectively, where $R$ indicates the distance of the impact location of the EAS from the center of FoV. The open circles and squares include a zenith angle cut of $\theta>60^{\circ}$.

\section{Geometrical aperture and cloud impact}

To estimate the geometrical aperture, a large number of EASs are simulated by uniformly injecting them over an extended area $S_{\text {inject }} \gg S_{\text {obs }}$ in a clear atmosphere condition for nominal background level of $I_{\mathrm{BG}}=500$ photons $\mathrm{m}^{-2}$ $\mathrm{ns}^{-1} \mathrm{sr}^{-1}$.

For $N_{\text {trig }}$, triggering samples among $N_{\text {inject }}$, simulated EAS events with an energy $E$, the corresponding geometrical aperture $A(E)$ is defined by the following relation:

$$
A(E)=\frac{N_{\text {trig }}}{N_{\text {inject }}} \cdot S_{\text {inject }} \cdot \Omega_{0}
$$

where $\Omega_{0}=\pi$ sr is the solid angle acceptance for $0^{\circ}<\theta<90^{\circ}$. As explained before, by applying simple cuts on the distance $R$ from the center of FoV of the impact location of the EAS, and on the lower limit $\theta_{\text {cut }}$, the geometrical aperture $A_{\text {sub }}$ is derived as follows:

$$
A_{\mathrm{sub}}(E)=2 \pi \int_{S_{\mathrm{sub}}} \int_{\theta_{\mathrm{cut}}}^{\pi} \epsilon(E, \theta, \vec{r}) \cdot \cos \theta \cdot \sin \theta d \theta d S,
$$

where $d S$ is the area element in the selected subsection of the observation area $S_{\mathrm{sub}}$, and $\epsilon(E, \theta, \vec{r})$ is the probability of trigger at the impact location $\vec{r}$ with respect to the center of FoV.

Figure 10 shows the geometrical aperture as a function of energy for $H_{0}=$ $400 \mathrm{~km}$ along with the apertures for different geometrical cuts in $\theta$ and $R$. Figure 11] shows the trigger efficiency as a function of core location for different cuts in $E$ and $\theta$. 

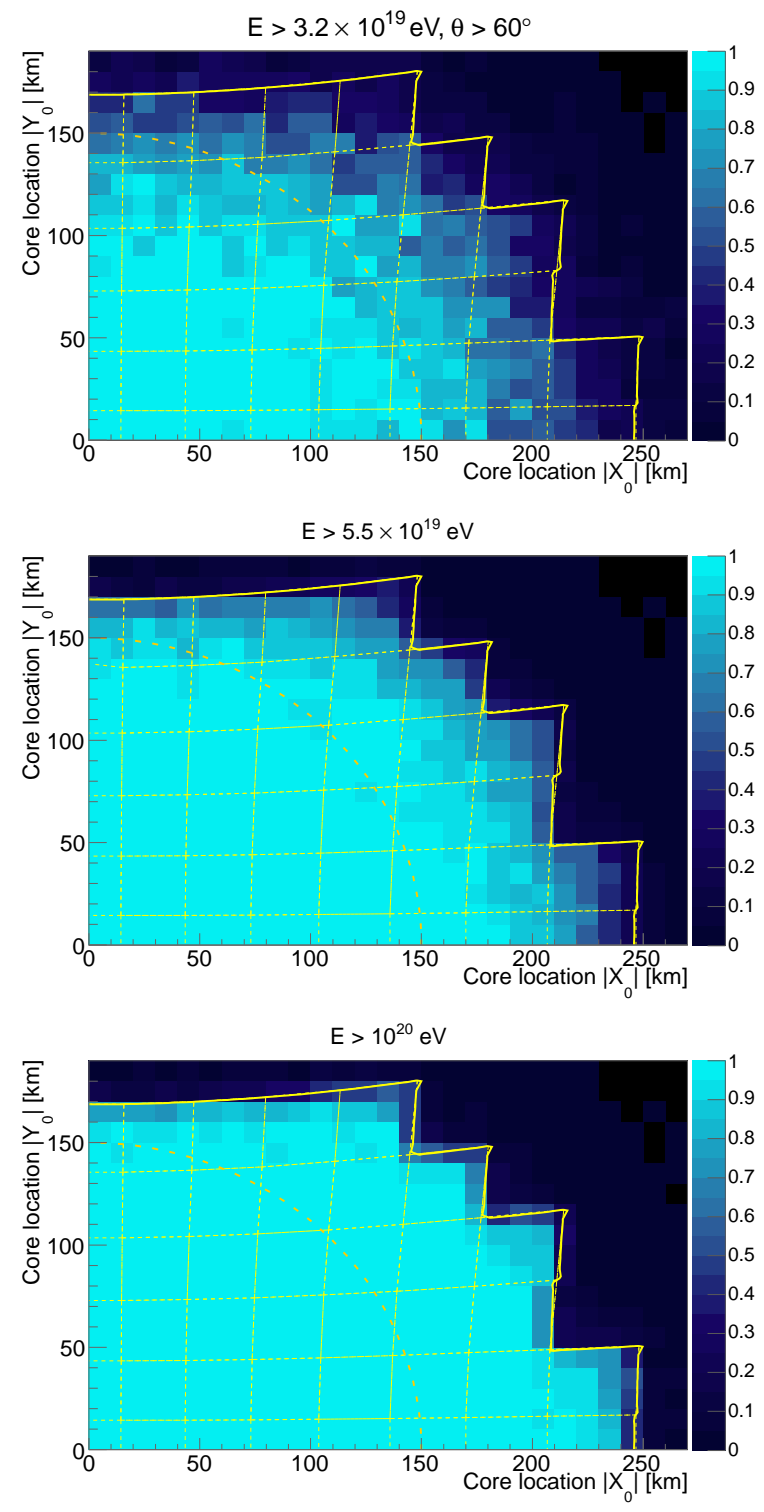

Figure 11: Trigger probability as a function of core location with different geometrical and energy cuts: top) $E>3.2 \times 10^{19} \mathrm{eV}$ with $\theta>60^{\circ}$; middle) $E>5.5 \times 10^{19} \mathrm{eV}$ and bottom) $E>10^{20} \mathrm{eV}$. The last two cases are with no zenith angle cuts.

The geometrical aperture without geometrical cut reaches the plateat ${ }^{3}$ above

\footnotetext{
${ }^{3}$ It is defined by the condition in which the geometrical aperture is $>0.8 \cdot S \cdot \Omega$ for the area
} 
$\sim(6-7) \times 10^{19} \mathrm{eV}$. At the highest energies, the geometrical aperture is close to saturation. The value is mainly determined by $S_{\text {obs }}$ for a given $H_{0}$ and, therefore, higher altitudes result in the larger saturating apertures. Due to a minor contribution of EAS crossing the FoV, the geometrical aperture grows slightly with energy.

By applying the cut $\theta>60^{\circ}$, which reduces the solid angle acceptance to $\pi / 4 \mathrm{sr}$, a constant aperture is achieved above $\sim(4-5) \times 10^{19} \mathrm{eV}$. In addition, a more stringent cut with $R<150 \mathrm{~km}$ extends the constant aperture range down to $\sim 3 \times 10^{19} \mathrm{eV}$. The possibility to extend the plateau region at lower energies for a subset of events will allow a cross-check of the flux measured by the full sample of events in the specific range of energies where the aperture of the instrument has not reached the plateau level yet. Consequently, the overlapping energy range between JEM-EUSO and ground-based observatories will be enlarged.

As mentioned in Section 4 the altitude of the EAS maximum $H_{\max }$ compared to that of the cloud-top is an important parameter to decide if the EAS properties are reconstructed in a sufficiently precise way in presence of clouds. Figure 12 represents $H_{\max }$ of triggered EASs in clear atmosphere as a function of zenith angle. $H_{\max }$ is strongly dependent on the zenith angle of the EAS. For proton EASs, $\sim 80 \%$ of the events have their maximum at altitudes higher than $3.2 \mathrm{~km}$, which is the value typically used in literature for the cloud-top altitude of low level clouds. It should be mentioned that the elongation rate of $X_{\max }$ (depth of shower maximum), i.e. $\partial X_{\max } / \partial \log E$ is $\sim 80 \mathrm{~g} \mathrm{~cm}^{-2}$ per energy decade leading to only a limited increase $\partial H_{\max } / \partial \log E \sim 1 \mathrm{~km}(\sim 0.3 \mathrm{~km})$ per decade for $\theta=0^{\circ}\left(60^{\circ}\right)$. For heavier particles, $H_{\max }$ is slightly higher, and, therefore, less affected by the presence of clouds.

In order to evaluate the effect of clouds on the trigger efficiency more precisely, EAS simulations for different cloudy cases are performed. Four cloud-top altitudes $H_{\mathrm{C}}=2.5,5,7.5$ and $10 \mathrm{~km}$ are considered, as well as four optical depths of the test cloud $\tau_{\mathrm{C}}=0.05,0.5,1.5$ and 5 . To quantify the effect of clouds, the ratio between the trigger aperture in a given cloudy condition to that of a clear atmosphere case, $\zeta_{\mathrm{C}}\left(E ; H_{\mathrm{C}}, \tau_{\mathrm{C}}\right)$, is calculated for each specific cloud condition $\left(H_{\mathrm{C}}, \tau_{\mathrm{C}}\right)$ as a function of $E$ :

$$
\zeta_{\mathrm{C}}\left(E ; H_{\mathrm{C}}, \tau_{\mathrm{C}}\right)=\frac{A\left(E ; H_{\mathrm{C}}, \tau_{\mathrm{C}}\right)}{A(E ; \text { clear })} .
$$

In Table 4, the average values $\zeta_{\mathrm{C}}\left(E ; H_{\mathrm{C}}, \tau_{\mathrm{C}}\right)$ are summarized for the different test clouds and showers simulated with energies above $6.3 \times 10^{19} \mathrm{eV}$ and an assumed differential flux of $d N / d E \propto E^{-3}$.

In case of optically thick clouds with $\tau_{\mathrm{C}} \geq 1$, the trigger efficiency depends on $H_{\mathrm{C}}$. High altitude clouds in particular absorb EAS signals emitted beneath the cloud and result in a significant lowering of the trigger efficiency. At middle

$\bar{S}$ and solid angle acceptance $\Omega$ defined by specific geometrical cuts. 


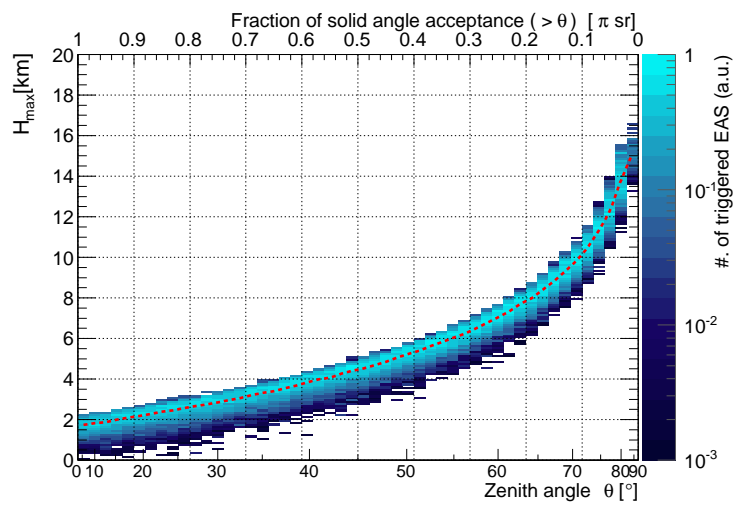

Figure 12: Distribution of the altitude of EAS maximum $H_{\max }$ as a function of zenith angle for triggered EAS events and an assumed differential flux of $d N / d E \propto E^{-3}$. The horizontal axis on the top shows the solid angle acceptance above the given zenith angle. This result is obtained by merging together all triggered events of all energies simulated in clear atmosphere conditions.

Table 4: Average $\zeta_{\mathrm{C}}$ values for different types of clouds and EASs simulated with energies above $6.3 \times 10^{19} \mathrm{eV}$ and an assumed differential flux of $d N / d E \propto E^{-3}$.

\begin{tabular}{l|cccc}
\hline \multirow{2}{*}{$\begin{array}{l}\text { Cloud-top } \\
\text { Altitude }\end{array}$} & \multicolumn{4}{|c}{ Optical depth $\tau_{\mathrm{C}}$} \\
\cline { 2 - 5 } & 0.05 & 0.5 & 1.5 & 5 \\
\hline$H_{\mathrm{C}}=10 \mathrm{~km}$ & $90 \%$ & $70 \%$ & $26 \%$ & $18 \%$ \\
$H_{\mathrm{C}}=7.5 \mathrm{~km}$ & $89 \%$ & $74 \%$ & $43 \%$ & $37 \%$ \\
$H_{\mathrm{C}}=5 \mathrm{~km}$ & $89 \%$ & $82 \%$ & $69 \%$ & $66 \%$ \\
$H_{\mathrm{C}}=2.5 \mathrm{~km}$ & $90 \%$ & $88 \%$ & $89 \%$ & $88 \%$ \\
\hline
\end{tabular}




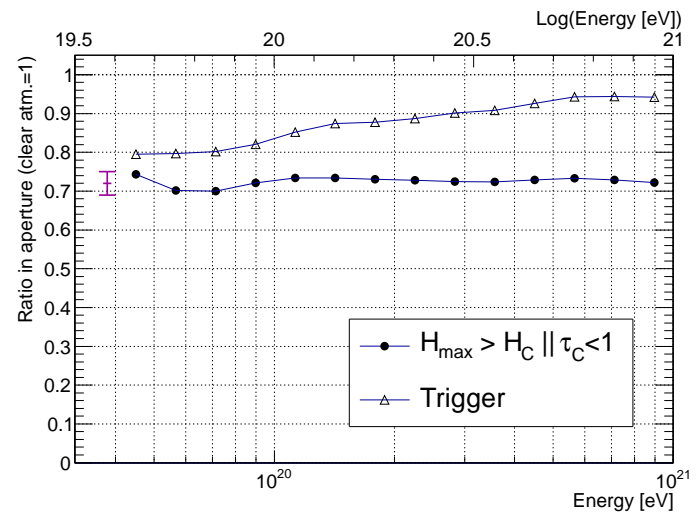

Figure 13: Relation between cloud efficiency and energy. The triangles show, as a function of energy, the results of the convolution of $\zeta_{\mathrm{C}}\left(E ; H_{\mathrm{C}}, \tau_{\mathrm{C}}\right)$ of Table 4 with the matrix of cloud occurrence of Table 3 Filled circles represent the case where we require $H_{\max }>H_{\mathrm{C}}$ for optically thick clouds. The cloud efficiency $\kappa_{\mathrm{C}}$ is defined as the average of the values expressed by the filled circles. The error bar on the left shows the uncertainty on the points $(\sim 3 \%)$.

altitudes, $H_{C} \sim 5 \mathrm{~km}$, clouds only influence EASs of small zenith angles, which develop at lower altitudes.

In the presence of high clouds with $\tau_{\mathrm{C}}<1$, the signal from an EAS below cloud level is only attenuated by a factor of $\exp \left(-\tau_{\mathrm{C}}\right)$ and the effect on the trigger efficiency is limited. If $H_{\mathrm{C}}$ is well below the altitudes where EAS develop, the clouds do not attenuate the signals. As a result of the different cuts, $\zeta_{\mathrm{C}}$ slowly increases with energy.

The results in Table 4 are then weighted with the relative cloud occurrence of Table 3 to estimate the average effect as a function of energy. The results are shown in Figure 13. For cloudy cases, the average of all triggered events is shown by triangles and it tends to increase with energy. The results obtained by applying the selection $H_{\max }>H_{\mathrm{C}}$ for $\tau_{\mathrm{C}}>1$ cases is indicated by filled circles. This cut requires that for optically thick clouds, the EAS maximum is located above the cloud-top. This ensures that fitting the EAS profile will not introduce significant distortion of the reconstructed EAS profile. With the above cut, the fraction of selected events over the ones triggering in clear-sky conditions (the reference case) is almost constant at higher energies. This is because a certain fraction of clouds with $\tau_{\mathrm{C}}>1$ exist at higher altitudes. From Table 3, for example, clouds with $H_{\mathrm{C}}>6.5 \mathrm{~km}$ account for $\sim 20 \%$ of cloud coverage. Therefore, a part of EAS develops below such clouds. As this value is nearly constant as a function of energy, we define it as the 'cloud efficiency' $\kappa_{\mathrm{C}}$ and it accounts for $\sim 72 \%$ of the trigger EASs above $\sim 3 \times 10^{19} \mathrm{eV}$. This is due to the fact that the average $H_{\max }$ dependence is dominated by the zenith angle. The energy plays a smaller role.

The value $\kappa_{\mathrm{C}} \sim 72 \%$ is an important factor for estimating the effective exposure of the mission. Currently, a detailed study on the reconstruction of 


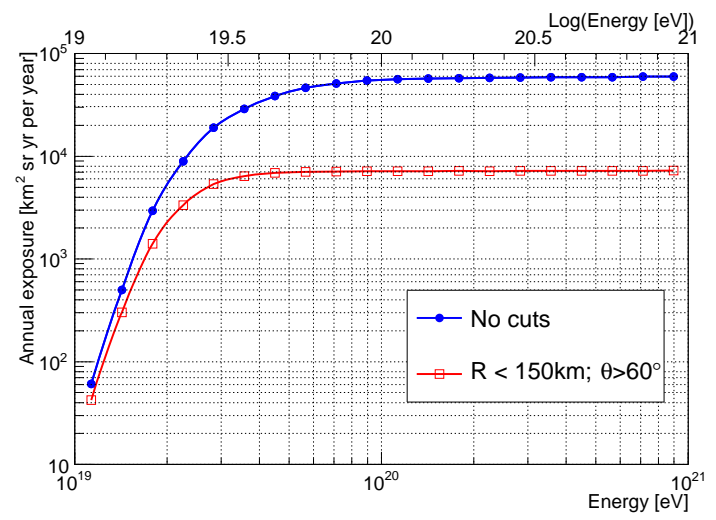

Figure 14: Annual exposure as a function of energy obtained for the two extreme conditions shown in Figure 10 a) entire observation area (filled circles); b) cut on distance $R<150 \mathrm{~km}$ and on zenith angle $\theta>60^{\circ}$ (open squares).

the events passing such a trigger selection as well as those occurring in a clear atmosphere is in progress. It should be emphasized that the main telescope of JEM-EUSO will be operated along with AM system [22, 55].

\section{Exposure}

From the above results, the exposure per year of operation for events that trigger JEM-EUSO, defined as the 'annual exposure' is evaluated as a function of energy:

$$
(\text { Annual exposure }) \equiv A(E) \cdot \kappa_{\mathrm{C}} \cdot \eta_{0} \cdot\left(1-f_{\mathrm{loc}}\right) \cdot(1[\mathrm{yr}])
$$

In this estimation, we use $\kappa_{\mathrm{C}}=72 \%, \eta_{0}=20 \%$, and $f_{\text {loc }}=10 \%$, respectively. The operational inefficiencies related to ISS (rockets docking on ISS, lid operation, detector maintenance or aging, etc.) as well as quality cuts on reconstruction are not taken into account yet, and will be addressed in future. Therefore, the present results constitute an upper limit on the effective exposure of the instrument for the assumed conditions.

In Figure 14 the annual exposure as a function of energy is shown. The filled circles indicate the geometrical aperture for the entire observation area. The open squares include a zenith angle cut of $\theta>60^{\circ}$ and a cut on distance of $R<150 \mathrm{~km}$. These two exposures correspond to the highest and lowest aperture curves of Figure 10.

The JEM-EUSO annual exposure for the full sample of data is expected to be $\sim 9$ times larger than that of the Pierre Auger Observatory with the corresponding annual exposure of about $7000 \mathrm{~km}^{2} \mathrm{sr} y$, at energies around $10^{20} \mathrm{eV}$. Because of the steeply rising aperture at lower energies, the subsets of data with reduced and flat exposure will be used to cross-check with measurements by other ground-based experiments down to $\sim(2-3) \times 10^{19} \mathrm{eV}$. It is important 


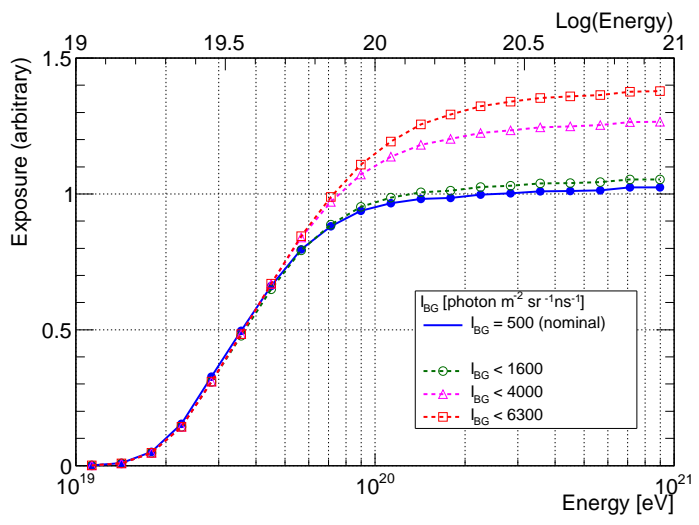

Figure 15: Exposure in arbitrary unit for different values of maximum acceptable background level.

to underline that the cuts shown in Figure 14 are extreme in order to obtain at $\sim 3 \times 10^{19} \mathrm{eV}$ an annual exposure comparable to that of Auger, which means acquiring a statistically similar data sample. As shown in Figure 10 for the apertures, less stringent geometrical cuts will lead to different exposure curves located in between the two lines shown in Figure 14 with a flat plateau starting gradually at higher energies. In this way, it will be possible to have a comparison of UHECR fluxes for one entire decade in energy using the data acquired without geometrical cuts.

It is worthwhile remembering here that the aperture and exposure have been derived with specific assumptions on the detector properties, background level, shower development in atmosphere, etc. All the systematic uncertainties that would increase or decrease the collected light at telescope level, either for the EAS or for the background, would be responsible to shift the energy scale of the aperture and exposure curves by the square root of the systematic uncertainty. On the other hand, the scaling factor would be linear in case it involves only the EAS propagation (i.e. hadronic interaction model, fluorescence yield).

In the previous analysis, a constant background level of $\left\langle I_{\mathrm{BG}}\right\rangle=500$ photons $\mathrm{m}^{-2} \mathrm{~ns}^{-1} \mathrm{sr}^{-1}$ was assumed. However, the background is variable with time. To take into account the effective background variation, the exposure over the time when $I_{\mathrm{BG}}<I_{\mathrm{BG}}^{\mathrm{thr}}$ given as a function of $E$ is approximated by the following relation:

$$
(\text { Overall exposure }) \propto \int_{0}^{I_{\mathrm{BG}}^{\mathrm{thr}}} A\left(\sqrt{\frac{\left\langle I_{\mathrm{BG}}\right\rangle}{I_{\mathrm{BG}}}} \cdot E\right) \cdot p\left(I_{\mathrm{BG}}\right) d I_{\mathrm{BG}} .
$$

Figure 15] shows the exposure as a function of $E$ for various maximum allowed background levels obtained by convolving the trigger probability at a specific fixed background level with the fraction of time during which such background level occurs according to the estimation by Equations (2) and (3). As described in Section [5, the trigger system is capable of dynamically adjusting the thresh- 


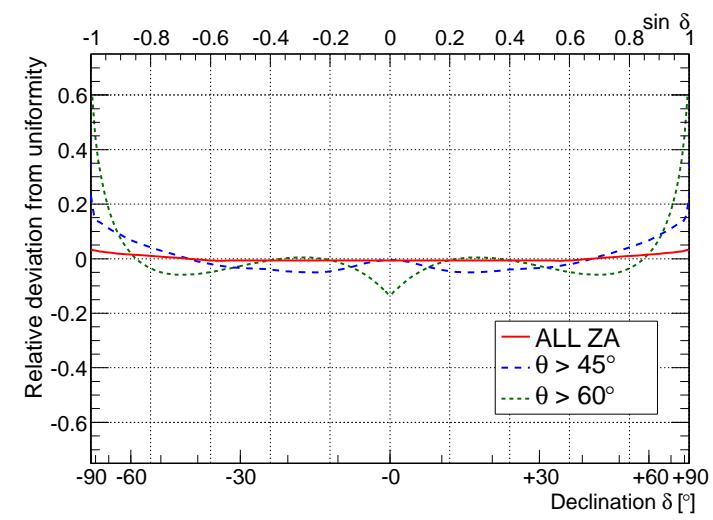

Figure 16: Expected distribution of observed exposure as a function of declination with different zenith angle cuts for all zenith angles (solid line), $\theta>45^{\circ}$ (dashed line) and $\theta>60^{\circ}$ (dotted line). The vertical axis indicates the deviation from the uniform distribution.

olds to cope with variable background intensity. The trigger efficiency curve scales in energy approximately in a proportional way to $\sqrt{I_{\mathrm{BG}}}$ because it depends on the Poissonian fluctuations of the average background level.

The exposure obtained with a fixed $I_{\mathrm{BG}}$ of 500 photons $\mathrm{m}^{-2} \mathrm{~ns}^{-1} \mathrm{sr}^{-1}$ is essentially equivalent to the one obtained from Equation (11) when the integration of $I_{\mathrm{BG}}$ is extended up to 1600 photons $\mathrm{m}^{-2} \mathrm{~ns}^{-1} \mathrm{sr}^{-1}$. It is possible to observe that at higher energies there is still some margin of gain if a higher level of background is accepted (see i.e. the curve at less than 6300 photons $\mathrm{m}^{-2} \mathrm{~ns}^{-1} \mathrm{sr}^{-1}$ ). This is particularly useful to explore the extreme energy ranges where the flux is rapidly decreasing with energy. In any case, all the conclusions obtained in this paper are derived assuming only the standard condition of 500 photons $\mathrm{m}^{-2} \mathrm{~ns}^{-1} \mathrm{sr}^{-1}$ constant background level.

Unlike ground-based observatories, the global ISS orbit and better sensitivities for EAS with large zenith angles allows observation of the entire Celestial Sphere. The exposure distribution is practically flat in right ascension. Apart from possible local or seasonal deviation from the global average of cloud coverage and of background level, the relationship between the expected overall exposure and declination can be analytically calculated as a function of only $\theta_{\text {cut }}$, knowing the observable nighttime at a given latitude.

Figure 16] shows the expected distribution of observed exposure as a function of declination on the celestial sphere with different zenith angle cuts for all zenith angles (solid line), $\theta>45^{\circ}$ (dashed line) and $\theta>60^{\circ}$ (dotted line). The vertical axis indicates the deviation from the uniform distribution.

For the case of $\theta>60^{\circ}$, minor excesses and deficits arise in very limited regions near Celestial Poles and Equator, respectively. This is because the ISS has a slightly longer residence time at high latitudes. JEM-EUSO can achieve in general a nearly constant exposure for the full range of zenith angles for which the arrival direction analysis will be performed. This is one of the advantageous 
features of space-based observation, as only small correction factors are needed.

\section{Discussion}

The convolution of observational duty cycle $\eta_{0} \sim 20 \%$, cloud efficiency $\kappa_{c}$ $\sim 72 \%$ and effect of local light $f_{\text {loc }} \sim 10 \%$ gives an overall conversion factor from geometric aperture to exposure of about $\sim 13 \%$ with slight variations depending on the cuts applied to the different terms. This is due to the particular features of observation from space: a) the possibility of operating in the presence of some diffuse moonlight up to $68 \%$ of the time, and b) the possibility of measuring in a substantial fraction of the solid angle acceptance even in presence of lowor middle- altitude clouds. As an example, the distribution of cloud occurrence around the location of Auger has been compared to the ISS case by using TOVS data though with statistical errors $\sim 6 \%$. Results show that, despite a significantly higher fraction of clear atmosphere at Auger site $(\sim 42 \%)$ compared to the world average (day and night) spanned by the ISS $(\sim 28 \%)$, when the fraction of time in which the cloud-top is located below $3.2 \mathrm{~km}$ is added to the clear atmosphere, both Auger and ISS orbit give similar results $(\sim 60 \%)$. For space-based observation this is the factor that should be compared with the clear atmosphere fraction of a ground-based observatory.

Our results are in agreement with those obtained by [48] for the observational duty cycle. Our result is slightly higher than what has been reported in [56] for the OWL observatory mainly due to the fact that OWL will fly at higher altitudes $(\sim 1000 \mathrm{~km})$, reducing the duty factor for full darkness by about $15 \%^{-}$ $20 \%$ due to the shorter nighttime. Moreover, in our estimation we have taken into account the possibility of accepting some moonlight as long as the total diffuse light is less than 1500 photons $\mathrm{m}^{-2} \mathrm{~ns}^{-1} \mathrm{sr}^{-1}$.

Concerning clouds, our efficiency is a factor of $\sim 1.5$ times larger than [48] essentially due to the fact that the cut on $H_{\max }$ is applied only to optically thick clouds. This is motivated by the fact that thin clouds might distort the shower profile but not affect the arrival direction of the primary particle and therefore may be used to perform anisotropy analyses.

Even though it is not considered in the present analysis, it is interesting to observe that optically thick clouds $\left(\tau_{\mathrm{C}}>2\right)$, located at low altitudes, may play another positive role, which is blocking the anthropogenic light and, therefore, allowing EAS measurement also in regions typically polluted by city light.

Our results do not support the conclusions in [57], where it was claimed that the sensitivity of space-based fluorescence detectors is of unacceptably small level. In that approach, only cloud-free scenes are considered and we know from Section 4 that they account for only $1 / 3$ of the time. Moreover, very strict conditions were applied on the extension of the cloud-free area. As an example, no correlation on the altitude of the possibly cloudy pixel and location of the track was applied. This tends to reject very inclined events with much longer paths in atmosphere even though all the detectable part of the track is located above clouds. It is clear that these constraints severely reduce the overall efficiency. The authors commented that less rigorous constraints could increase 
the cloud-free efficiency by even a factor of 3 . Finally, as previously mentioned, simulations have proved [7, 40] the feasibility of reconstructing EAS with reasonable uncertainty in presence of clouds. It is important to stress that the AM system will have an important role in monitoring the atmospheric conditions in which EAS develop, together with information from satellites, ground-based observations and meteorological models. We are, therefore, confident that it will be possible to characterize the atmospheric conditions in which each EASs has been detected, and, account for these conditions in the data analyses. Finally, it has to be mentioned that most of the above problems are related to the estimation of the energy and $X_{\max }$ of the EAS. The estimation of the exposure is more easily assessed and does not depend strongly on the level of accuracy of the EAS reconstructed parameters.

The present results indicate that JEM-EUSO has the potential to reach an annual exposure of nearly one order of magnitude higher than Auger at energies around $10^{20} \mathrm{eV}$. However, a final assessment of the annual exposure requires an evaluation of event selection efficiency to ensure the quality of the reconstructed EASs in terms of energy, angular resolution, and $X_{\max }$. This will be described in detail in a forthcoming paper. Preliminary results (see [58]), assuming clear atmosphere indicate that this condition is satisfied by most of the events.

It is possible that the full data sample will be subdivided in sub-samples of data peculiar for each analysis. As an example, those events reconstructed in thin cloud conditions are still most probably usable for the anisotropy analyses, granted that a lower limit on the energy of the event is assigned with high confidence. Moreover, the possibility of defining a sample of events detected in 'golden conditions' such as inclined EASs in clear-atmosphere will guarantee a cross-check of the reliability of wider samples of events.

It will be possible to significantly increase the exposure by tilting the telescope. In the tilt mode, the observation area is scaled by $\sim(\cos \xi)^{-3}$ as a function of titling angle $\xi$ of the optical axis from the nadir. This will increase the sample at the highest energies and help to compensate the reduction of the observation area in case of periods of lower orbiting altitude. As an example, in case of $H_{0} \sim 350 \mathrm{~km}$, tilting the instrument by $\xi \sim 25^{\circ}$ would give an observation area similar to the case of $H_{0} \sim 400 \mathrm{~km}$ in nadir mode. At the same time, the observation in nadir mode would extend by $\sim 30 \%$ the lowest energies where the measurement would be feasible. The analyses in such 'quasi-nadir mode' in which the optical axis is tilted by $\sim 0^{\circ}-25^{\circ}$, can be easily assimilated to the nadir one. In case of even larger tilting angles $\left(\xi \geq 25^{\circ}\right)$, a dedicated study is necessary to evaluate the performance. This will be addressed in future.

We wish also to point out that JEM-EUSO has considerably improved with respect to the original Extreme Universe Space Observatory [59] that successfully completed Phase-A study within ESA. The main improvements can be ascribed to the baseline optics of the JEM-EUSO telescope [18] (with $\sim 1.5$ better focusing capability), to the FS detector [19] ( 1.6 higher detection efficiency), to the better geometrical layout of PDMs on the FS that maximizes the filling factor [21], and to the improved performance of the electronics [60, 46], which allow more complex trigger algorithms [61]. 


\section{Summary}

The most important factors which determine the annual exposure of JEMEUSO mission have been reviewed. The analytical calculations indicate that the operational duty cycle of JEM-EUSO, or the fraction of time in which the EAS measurement is not hampered by the brightness of the atmosphere, is of the order of $\eta_{0} \sim 20 \%$. The local light such as city light, atmospheric flashes and auroras will reduce the effective instantaneous observational area to $1-f_{\text {loc }} \sim 90 \%$ of the geometrical area. The role of clouds has been thoroughly investigated and the cloud efficiency, defined as the ratio of the effective average aperture to the geometrical aperture, is found to be $\kappa_{c} \sim 72 \%$. All the above factors give an overall conversion factor from geometric aperture to exposure of about $\sim 13 \%$. Simulations show that JEM-EUSO can reach almost full efficiency at energies around $3 \times 10^{19} \mathrm{eV}$ for a restricted subset of events, and for the full aperture at energies $E \gtrsim(6-7) \times 10^{19} \mathrm{eV}$. The expected annual exposure of JEM-EUSO around $10^{20} \mathrm{eV}$ is equivalent to about 9 years exposure of Auger. This value has to be presented as the potential of JEM-EUSO in nadir mode. A study of the selection efficiency of events due to quality cuts on reconstruction in clear and cloudy conditions will be performed in future to refine these results.

\section{Acknowledgments}

This work was partially supported by Basic Science Interdisciplinary Research Projects of RIKEN and JSPS KAKENHI Grant (22340063, 23340081, and 24244042), by the Italian Ministry of Foreign Affairs, General Direction for the Cultural Promotion and Cooperation, by the 'Helmholtz Alliance for Astroparticle Physics HAP' funded by the Initiative and Networking Fund of the Helmholtz Association, Germany, and by Slovak Academy of Sciences MVTS JEM-EUSO as well as VEGA grant agency project 2/0081/10. The Spanish Consortium involved in the JEM-EUSO Space Mission is funded by MICINN under projects AYA2009-06037-E/ESP, AYA-ESP 2010-19082, AYA2011-29489C03-01, AYA2012-39115-C03-01, CSD2009-00064 (Consolider MULTIDARK) and by Comunidad de Madrid (CAM) under project S2009/ESP-1496.

The many constructive comments and suggestions of two anonymous referees are acknowledged.

\section{References}

[1] M. Takeda et al., Astrophys. J. 522 (1999) 255.

[2] R. Abbasi et al., Astropart. Phys. 30 (2008) 175.

[3] The Pierre Auger Collaboration, Science 318 (2007) 938.

[4] K. Greisen, Phys. Rev. Lett. 16 (1966) 748.

[5] G.T. Zatsepin and V.A. Kuz'min, JETP Lett. 4 (1966) 78. 
[6] T. Ebisuzaki et al., Nucl. Phys. B (Proc. Suppl.) 175 (2008) 237.

[7] Y. Takahashi et al., New J. Phys. 11 (2009) 065009.

[8] M. Casolino et al., Astrophys. Space Sci. Trans 7 (2011) 477.

[9] NASA, ISS User's Guide-Release 2.0, 2000.

[10] J.N. Matthews et al., Nucl. Phys. B (Proc. Suppl.) 212 (2011) 79.

[11] V. P. Egorova et al., Nucl. Phys. B, Proc. Suppl. 136 (2004) 3.

[12] J. Abraham et al., Nucl. Instr. and Meth. A 523 (2004) 50.

[13] JAXA Human Space Systems and Utilization Program Group, Kibo Handbook, 2007.

[14] G. Medina-Tanco et al., Proc. 32 ${ }^{\text {nd }}$ Int. Cosmic Ray Conf., Beijing (2011) Vol.3, p.204; (preprint) arXiv:1204.5065

[15] R.M. Baltrusaitis et al., Nucl. Instr. and Meth. A 240 (1985) 410.

[16] T. Abu-Zayyad et al., Nucl. Instr. and Meth. A 450 (2000) 253.

[17] F. Kajino et al., Nucl. Inst. Meth. A 623 (2010) 422.

[18] A. Zuccaro Marchi et al., Proc. $32^{\text {nd }}$ Int. Cosmic Ray Conf., Beijing (2011) Vol.3, p.176; (preprint) arXiv:1204.5065

[19] Y. Kawasaki et al., Proc. 32 ${ }^{\text {nd }}$ Int. Cosmic Ray Conf., Beijing (2011) Vol.3, p.96; (preprint) arXiv:1204.5065.

[20] M. Casolino et al., Proc. 32 ${ }^{\text {nd }}$ Int. Cosmic Ray Conf., Beijing (2011) Vol.3, p.290; (preprint) arXiv:1204.5065

[21] M. Ricci et al., Proc. 32 ${ }^{\text {nd }}$ Int. Cosmic Ray Conf., Beijing (2011) Vol.3, p.80; (preprint) arXiv:1204.5065.

[22] A. Neronov et al., Proc. 32 ${ }^{\text {nd }}$ Int. Cosmic Ray Conf., Beijing (2011) Vol.6, p.332; (preprint) arXiv:1204.5065

[23] F. Arqueros, J.R. Hörandel and B. Keilhauer, Nucl. Instr. Meth. A 597 (2008) 1.

[24] G. Lefeuvre et al., Nucl. Instr. Meth. A 578 (2007) 78.

[25] http://www.spaceref.com/news/viewsr.html?pid=12848

[26] http://spaceflight.nasa.gov/station/flash/iss_attitude.html

[27] K. Higashide et al., Proc. $32^{\text {nd }}$ Int. Cosmic Ray Conf., Beijing (2011) Vol.3, p.294; (preprint) arXiv:1204.5065 
[28] D. Supanitsky et al., Proc. 31 ${ }^{\text {st }}$ Int. Cosmic Ray Conf., Lodz (2009) \#1424.

[29] http://sscweb.gsfc.nasa.gov/cgi-bin/Locator.cgi

[30] F. Montanet, Internal note of EUSO Collaboration, EUSO-SIM-REP-009$1.2(2004)$.

[31] N. Cox, Allen's Astrophysical Quantites, 4th Edition, Springer-Verlas, New York 2000.

[32] K. Krisciunas and B.E. Schaefer, Publ. Atron. Soc. of the Pacific 103 (1991) 103 .

[33] A.-M. Frida et al., Tellus A 58/3 (2006) 320.

[34] R.B.A. Koelemeijer et al., Remote Sensing of Environment 63/3 (1998) 279.

[35] G.K. Garipov et al., Astropart. Phys. 24 (2005) 400.

[36] P. Barbier et al., Astropart. Phys. 22 (2005) 439.

[37] O. Catalano et al., Nucl. Instr. Meth. A 480 (2002) 547.

[38] http://www.swpc.noaa.gov/Aurora/index.html

[39] http://www.ngdc.noaa.gov/dmsp/

[40] T. Abu-Zayyad et al., Astropart. Phys., 21 (2004) 163.

[41] F. Fenu et al., Proc. 32 ${ }^{\text {nd }}$ Int. Cosmic Ray Conf., Beijing (2011) Vol.3, p.116; (preprint) arXiv:1204.5065,

[42] http://www.ozonelayer.noaa.gov/action/tovs.htm/

[43] http://isccp.giss.nasa.gov/

[44] http://www.atmos.washington.edu/CloudMap/

[45] F. Garino, Master Thesis, University of Torino, Italy (2010).

[46] J. Bayer et al., Proc. 32 ${ }^{\text {nd }}$ Int. Cosmic Ray Conf., Beijing (2011) Vol.3, p.168; (preprint) arXiv:1204.5065.

[47] O. Catalano et al., Proc. $31^{\text {st }}$ Int. Cosmic Ray Conf., Lodz (2009), \#0326.

[48] C. Berat et al., Astropart. Phys. 33 (2010) 221.

[49] N.P. Ilina et al., Sov. J. Nucl. Phys. 55 (1992) 1540.

[50] D. Heck et al., Report FZKA \#6019 (1998).

[51] N.N. Kalmikov et al., Phys. Atom. Nucl. 56 (1993) 346. 
[52] M. Nagano et al., Astropart. Phys. 22 (2004) 235.

[53] F.X. Kneizys et al., User's Guide to LOWTRAN 7, AFGL-TR-0177, U.S. Air Force Geophysics Laboratory, Hanscom (1988).

[54] http://www.schott.com/advanced_optics/english/download/schott_bandpass_bg3_2008_e.pdf

[55] J.A. Morales et al., Proc. 32 ${ }^{\text {nd }}$ Int. Cosmic Ray Conf., Beijing (2011) Vol.11, p.466; (preprint) arXiv:1204.5065

[56] J.W. Mitchell et al., Proc. $31^{\text {st }}$ Int. Cosmic Ray Conf. Lodz (2009), \#1440.

[57] P. Sokolsky and A. Krizmanic, Astropart. Phys. 20 (2004) 391.

[58] M. Bertaina et al., Proc. $32^{\text {nd }}$ Int. Cosmic Ray Conf., Beijing (2011) Vol.3, p.219; (preprint) arXiv:1204.5065, M. Bertaina et al., Proc. UHECR2012, in press.

[59] The EUSO Collaboration, EUSO: Report on the Phase A Study, internal note EUSO-PI-REP-002 (2003).

[60] I.H. Park et al., Proc. 32 ${ }^{\text {nd }}$ Int. Cosmic Ray Conf., Beijing (2011) Vol.3, p.301; (preprint) arXiv:1204.5065.

[61] M. Bertaina et al., Proc. $30^{\text {th }}$ Int. Cosmic Ray Conf., Merida (2007) Vol.5, p.1049. 